\title{
Gluino meets flavored naturalness
}

\author{
Monika Blanke, ${ }^{a, b}$ Benjamin Fuks, ${ }^{c, d}$ Iftah Galon ${ }^{e}$ and Gilad Perez ${ }^{f}$ \\ ${ }^{a}$ Institut fur Theoretische Teilchenphysik, Karlsruhe Institute of Technology, \\ Engesserstraße 7, D-76128 Karlsruhe, Germany \\ ${ }^{b}$ Institut fur Kernphysik, Karlsruhe Institute of Technology, \\ Hermann-von-Helmholtz-Platz 1, D-76344 Eggenstein-Leopoldshafen, Germany \\ ${ }^{c}$ Sorbonne Universités, UPMC Univ. Paris 06, \\ UMR 7589, LPTHE, F-75005, Paris, France \\ ${ }^{d}$ CNRS, UMR 7589, LPTHE, \\ F-75005, Paris, France \\ ${ }^{e}$ Department of Physics and Astronomy, \\ University of California, Irvine, CA 92697, U.S.A. \\ ${ }^{f}$ Department of Particle Physics and Astrophysics, \\ Weizmann Institute of Science, Rehovot 76100, Israel \\ E-mail: monika.blanke@kit.edu, fuks@lpthe.jussieu.fr, iftachg@uci.edu, \\ gilad.perez@weizmann.ac.il
}

ABSTRACT: We study constraints from LHC run I on squark and gluino masses in the presence of squark flavor violation. Inspired by the concept of 'flavored naturalness', we focus on the impact of a non-zero stop-scharm mixing and mass splitting in the right-handed sector. To this end, we recast four searches of the ATLAS and CMS collaborations, dedicated either to third generation squarks, to gluino and squarks of the first two generations, or to charm-squarks. In the absence of extra structure, the mass of the gluino provides an additional source of fine tuning and is therefore important to consider within models of flavored naturalness that allow for relatively light squark states. When combining the searches, the resulting constraints in the plane of the lightest squark and gluino masses are rather stable with respect to the presence of flavor-violation, and do not allow for gluino masses of less than $1.2 \mathrm{TeV}$ and squarks lighter than about $550 \mathrm{GeV}$. While these constraints are stringent, interesting models with sizable stop-scharm mixing and a relatively light squark state are still viable and could be observed in the near future.

KEYwords: Beyond Standard Model, Supersymmetric Standard Model

ARXIV EPRINT: 1512.03813 


\section{Contents}

1 Introduction 1

2 Theoretical framework: a simplified model for studying gluino flavor violation

3 Monte Carlo simulations and LHC analysis reinterpretation details

3.1 Technical setup and general considerations 5

3.2 ATLAS: multijets $+\mathbb{E}_{T}+$ lepton veto 6

3.3 CMS: single lepton + at least four jets (including at least one $b$-jet) $+\mathbb{E}_{T} \quad 7$

3.4 CMS: at least 3 jets $+\mathbb{E}_{T}+$ lepton veto 8

3.5 ATLAS: scharm pair-production using charm-tagging + lepton veto 8

4 Results $\quad 9$

4.1 ATLAS: multijets $+\mathbb{E}_{T}+$ lepton veto 10

4.2 CMS: single lepton + at least four jets (including at least one $b$-jet) $+\mathbb{E}_{T} \quad 13$

$\begin{array}{lll}4.3 & \text { CMS: at least } 3 \text { jets }+\mathbb{E}_{T}+\text { lepton veto } & 13\end{array}$

4.4 ATLAS: scharm pair-production using charm-tagging + lepton veto 14

$\begin{array}{lll}4.5 & \text { Combined reach } & 18\end{array}$

5 Conclusions $\quad 22$

A Implementation and validation of ATLAS-SUSY-2013-04 in MADANALYSIS 5

\section{Introduction}

While the Large Hadron Collider (LHC) has just begun its second period of data taking, its first run has been an experimental success with many new measurements at an energy regime unexplored beforehand. In particular, the search for new phenomena has been given a boost with the discovery of a new particle, the celebrated Higgs boson $[1,2]$. Theoretically, however, we are still in the dark, as most searches performed at the LHC seem to be consistent with the Standard Model (SM) predictions. This includes the measurements of the Higgs couplings [3], the searches for new physics at the energy frontier with the ATLAS and CMS experiments and at the luminosity frontier with the LHCb experiment. ${ }^{1}$ In the absence of new physics signals, the naturalness argument that motivates the possible observation of new dynamics at the $\mathrm{TeV}$ scale seems slightly less appealing as a guiding

\footnotetext{
${ }^{1}$ Although the recent discovery of pentaquark-like states [4] is explained within the SM, the recent LHCb flavor anomalies might imply new physics [5-7].
} 
principle (see ref. [8] for a general status review and ref. [9] for a focus on supersymmetry). One of the main tasks of the next high-energy LHC runs at $13 \mathrm{TeV}$ and $14 \mathrm{TeV}$ will hence be to shed more light on the electroweak symmetry breaking mechanism and to estimate to what extent the Higgs-boson mass is fine-tuned.

One of the robust features of all natural extensions of the SM is the presence of top partners. These act to screen away the quadratic sensitivity of the Higgs-boson mass to the ultraviolet (UV) scales due mostly to the large top Yukawa coupling. Naively, one might expect that flavor physics and naturalness are two decoupled concepts. However, even within a minimal top partner sector, the definition of the flavor structure of the model could be non-trivial. The mass-eigenstates of the theory could be non-pure top partners and still yield a sufficient cancellation of the UV-sensitive quantum contributions to the Higgsboson mass. In this way, even a model exhibiting a single top-partner might incorporate large flavor- and CP-violating effects. This possibility, however, is typically ignored, due to prejudices and a possibly too simplistic interpretation of the bounds stemming from lowenergy flavor-changing neutral current processes. Indeed, most studies on naturalness have assumed either flavor universality among the partners or an approximate $\mathrm{U}(2)$ symmetry which acts on the partners of the first two generations. Nonetheless, a thorough analysis of the constraints arising from $D-\bar{D}$ and $K-\bar{K}$ mixing has shown that the degeneracy of the partners is not required for models of down alignment [10], and such frameworks in which new physics couplings are non-diagonal in flavor-space have been considered both in the context of supersymmetry [11, 12] and Higgs compositeness [13, 14].

Taking supersymmetry as an illustrative example, the non-degeneracy of the partners is even more appealing as the direct experimental bounds on second generation squarks are rather weak, their masses being only constrained to be larger than $500 \mathrm{GeV}$. This is a consequence of the underlying ingredients of all supersymmetry searches which are mainly sensitive either to 'valence' squarks or to third generation squarks [15]. If the supersymmetric top-partners are not flavor-eigenstates but rather admixtures of stops and scharms, then the signatures of supersymmetric events could change dramatically. In particular, the typically sought signatures such as top-quark pairs and missing transverse energy $\left(\mathbb{E}_{T}\right)$ could be exchanged for charm-jet pairs and top-charm pairs plus $\mathbb{E}_{T}$. This has led to the concept of supersymmetric 'flavored naturalness'. Despite the non-trivial flavor structure of the top sector, the level of fine tuning of these setups is similar to more conventional supersymmetric scenarios with pure-stop mass eigenstates and sometimes even improved [16]. In addition, it has been shown that low-energy electroweak and flavor physics still allow for large deviations from a minimal flavor structure in the squark sector [17-28].

Complementary, the gluino state included in any supersymmetric extension of the SM leads to an independent source of fine tuning [29]. This result is a combination of the rather strong bounds on the gluino mass, and the genuine gluino loop-diagram contributions to all scalar masses (and in particular to the squark masses $m_{\tilde{q}}$ ). Indeed, the gluino mass $m_{\tilde{g}}$ which is constrained to be larger than about $1 \mathrm{TeV}$, and even more in some specific setups, implies a naturalness relation between the squark and gluino masses,

$$
m_{\tilde{g}} \lesssim 2 m_{\tilde{q}}
$$


In this paper we focus on gluino phenomenology and study how the presence of a not that heavy gluino in the theory can give rise to bounds on flavored naturalness, and on non-minimal flavor mixing in the squark sector. In the case of squark flavor-violation, several new supersymmetric decay channels become relevant and new signatures could be expected. Although there is no specific experimental search dedicated to this nonminimally flavor-violating supersymmetric setup, the panel of signatures that can arise is large enough such that we can expect to be able to derive constraints from standard analyses that have been designed from flavor-conserving supersymmetric considerations, as already depicted in previous prospective studies [16, 19, 21-23, 28, 30-37]. We therefore investigate how standard searches for squarks and gluinos can be sensitive to a non-trivial flavor structure in the squark sector. In particular, we focus on four searches of the ATLAS and CMS collaborations, dedicated either to third generation squarks [38], to gluino and squarks from the first two generations [39, 40], or to charm-squarks [41]. This is only a representative subset of the vast experimental wealth of searches, but it is sufficient to derive meaningful results that constrain flavored naturalness since it covers all topologies that can arise in our non-minimally flavor-violating setup.

This work is structured as follows: the simplified model description that has been used throughout this analysis is introduced in section 2. Section 3 describes the reinterpretation procedure including the simulation setup and a concise summary of the experimental searches under consideration. The results are discussed in section 4, while our conclusions are presented in section 5. Details regarding the implementation of the ATLAS-SUSY-201304 search in the reinterpretation framework that we have used are provided in appendix A, while those related to the other considered searches can be found in ref. [42]

\section{Theoretical framework: a simplified model for studying gluino flavor violation}

Following a simplified model approach such as those traditionally employed by the ATLAS and CMS collaborations [43, 44], we consider a supersymmetric extension of the SM where only a subset of the superpartners feature masses accessible at the LHC. Effectively, we supplement the SM by a neutralino state $\tilde{\chi}^{0}$ which is the Lightest Supersymmetric Particle (LSP), one gluino state $\tilde{g}$ and two up-type squark states $\tilde{u}_{1}$ and $\tilde{u}_{2}$. The latter are mass eigenstates which are linear combinations of the right-handed stop and scharm flavor-eigenstates,

$$
\left(\begin{array}{c}
\tilde{u}_{1} \\
\tilde{u}_{2}
\end{array}\right)=\left(\begin{array}{rr}
\cos \theta_{23}^{R} & \sin \theta_{23}^{R} \\
-\sin \theta_{23}^{R} & \cos \theta_{23}^{R}
\end{array}\right)\left(\begin{array}{c}
\tilde{c}_{R} \\
\tilde{t}_{R}
\end{array}\right) \quad \text { with } \quad 0 \leq \theta_{23}^{R} \leq \pi / 4 .
$$

Note that the squark mixing angle lies in the $[0, \pi / 4]$ range so that the $\tilde{u}_{1}\left(\tilde{u}_{2}\right)$ state always contains a dominant scharm (stop) component. Throughout our analysis, we consider an extremely light neutralino with a mass fixed at $m_{\tilde{\chi}^{0}}=1 \mathrm{GeV}$. This assumption maximizes the amount of missing energy to be produced in signal events, and thus represents a favored scenario for any analysis relying on large missing energy signatures. Consequently, 
the results of this work represent a conservative estimate of the LHC sensitivity to the studied setup.

We define our model parameter-space by the three remaining masses, namely the squark and gluino masses $m_{\tilde{u}_{1}}, m_{\tilde{u}_{2}}$ and $m_{\tilde{g}}$, and the squark mixing angle $\theta_{23}^{R}$. Although strong bounds on the parameter-space could be derived from flavor physics observables even with just two 'active' squark states, all flavor constraints are, in practice, only sensitive to the quantity

$$
\left(\delta_{23}^{u}\right)_{R R} \approx\left|\frac{m_{\tilde{u}_{1}}-m_{\tilde{u}_{2}}}{m_{\tilde{u}_{1}}+m_{\tilde{u}_{2}}}\right|^{2} \sin \left(2 \theta_{23}^{R}\right)
$$

in the mass insertion approximation $[45,46]$ that is suitable for relatively small mass splittings and mixings. Since flavor bounds on the squark masses and mixings in the rightright sector are mild and arise mostly from $D$ physics [47, 48], flavor violation involving the right-handed stop and scharm is essentially unconstrained by flavor data, as long as the mixing between the stop and the up squark is assumed to be small. Therefore the squark mass difference $\Delta m=m_{\tilde{u}_{2}}-m_{\tilde{u}_{1}}$ and the sine of the mixing angle $\sin \theta_{23}^{R}$ could both be large, with related implications on squark pair-production cross sections [19]. Furthermore, LHC searches are potentially sensitive to the $\Delta m$ and $\sin \theta_{23}^{R}$ parameters separately, in contrast to flavor constraints which cannot disentangle the two [49]. ${ }^{2}$

The parameter-space can be divided into two domains depending on whether $\tilde{u}_{1}$ or $\tilde{u}_{2}$ is the lightest squark. For the sake of a clearer discussion and in order to put these two setups on an equal footing, we define two series of scenarios, both parameterized by a $\left(m_{\tilde{g}}, m_{\tilde{u}}, \Delta m, \theta_{23}^{R}\right)$ tuple, and distinguished by the sign of $\Delta m$. For the first series of scenarios (that we denote by S.I), the $m_{\tilde{u}}$ parameter is identified with the $\tilde{u}_{1}$ mass, while for the second series of scenarios (that we denote by S.II), the two squark masses are interchanged and the stop-dominated $\tilde{u}_{2}$ squark is now the lightest squark state with a mass given by $m_{\tilde{u}}$. This can be summarized, for given values of $\theta_{23}^{R}$ and $m_{\tilde{g}}$, as

$$
\left\{\begin{array} { l } 
{ \text { Scenarios of type S.I } } \\
{ m _ { \tilde { u } } = m _ { \tilde { u } _ { 1 } } < m _ { \tilde { u } _ { 2 } } } \\
{ \Delta m = m _ { \tilde { u } _ { 2 } } - m _ { \tilde { u } _ { 1 } } > 0 }
\end{array} \quad \text { and } \quad \left\{\begin{array}{l}
\text { Scenarios of type S.II } \\
m_{\tilde{u}}=m_{\tilde{u}_{2}}<m_{\tilde{u}_{1}} \\
\Delta m=m_{\tilde{u}_{2}}-m_{\tilde{u}_{1}}<0
\end{array} .\right.\right.
$$

In order to study the gluino effects on the constraints that can be imposed on flavored naturalness models, we perform a scan of the above parameter-space. The range in which each physical parameter of the model description is allowed to vary is given in table 1 . The $\Delta m=0$ case deserves a clarification. If the two squarks are indeed entirely degenerate, then the mixing can be rotated away as a consequence of the $\mathrm{U}(2)$ symmetry of the two squark mass-squared matrix. In this case, the mixing has thus no physical meaning. By imposing $\Delta m=0$, we in fact mean that the splitting between the squark states does not manifest itself in LHC processes and is still larger than the width of the squarks so that oscillation and interference effects are unimportant.

\footnotetext{
${ }^{2}$ This was pointed out in ref. [49] in the context of simplified models featuring a flavorful slepton sector. In practice, due to the highly restrictive lepton flavor bounds, their analysis included scenarios where only one parameter $\left(\Delta m\right.$ or $\left.\sin \theta_{23}^{R}\right)$ was large enough to induce an observable effect in the slepton searches of the first LHC run.
} 


\begin{tabular}{|c|c|c|c|}
\hline Parameter & Minimum value & Maximum value & Step \\
\hline$m_{\tilde{\chi}}[\mathrm{GeV}]$ & 1 & 1 & 0 \\
\hline$m_{\tilde{g}}[\mathrm{GeV}]$ & 800 & 2000 & 100 \\
\hline$m_{\tilde{u}}[\mathrm{GeV}]$ & 400 & $m_{\tilde{g}}$ & 100 \\
\hline$\Delta m[\mathrm{GeV}]$ & -500 & 500 & 100 \\
\hline$\theta_{23}^{R}$ & 0 & $\pi / 4$ & $\pi / 20$ \\
\hline
\end{tabular}

Table 1. Summary of the parameter-space of our simplified model. We present each physical parameter together with details associated with the scan that we have performed.

\section{Monte Carlo simulations and LHC analysis reinterpretation details}

\subsection{Technical setup and general considerations}

To determine the LHC sensitivity to the class of models introduced in section 2, we reinterpret the results of several ATLAS and CMS searches for supersymmetry for each point of the parameter-space scan defined in table 1. Technically, we have started by implementing the simplified model described above into FEYNRules [50], exported the model information in the UFO format [51] and have then made use of the MADGraph5_aMC@NLO [52] framework for event generation. The description of the QCD environment (parton showering and hadronization) has been achieved with the use of the PyтнiA 6 [53] package. Next, we apply a detector response emulator to the simulation results by means of the DeLPhes 3 program [54], that internally relies on the anti- $k_{T}$ jet algorithm [55] as implemented in the FASTJET software [56] for object reconstruction. For each of the recasted analyses, the DeLPHes configuration has been consistently tuned to match the setup depicted in the experimental documentation [42]. Finally, we have used the MADANALYsis 5 framework $[57,58]$ to calculate the signal efficiencies for the different search strategies, and to derive 95\% confidence level (CL) exclusions with the CLs method [59].

As most of the LHC analyses under consideration rely on a proper description of the jet properties, we have merged event samples containing up to one extra jet compared to the Born process and accounted for the possible double-counting arising from radiation that could be described both at the level of the matrix element and at the level of the parton showering by means of the Mangano (MLM) scheme [60, 61]. Moreover, we have normalized the cross-section for the signal samples to the next-to-leading-order (NLO) accuracy in QCD. Nevertheless, as flavor effects on supersymmetric production crosssections at NLO have yet to be calculated, we have taken a very conservative approach and applied a global $K$-factor of 1.25 to all leading-order results that have been obtained with MADGRAPH5_aMC@NLO.

In the above simulation chain, we employ a detector simulation which is much simpler than those of the CMS and ATLAS experiments. It cannot therefore genuinely account for the full complexity of the real detectors. For instance, event cleaning requirements or basic object quality criteria cannot be implemented in DeLPHES. While those are expected to only have a small impact on the limits that are derived, it is important to bear in 
mind that related uncertainties exist. Furthermore, the searches we are focusing on are multichannel searches, and the experimental limits are often derived after combining all channels. The statistical models that are used in the official exclusions are however not publicly available, so that we have made the approximation of considering each search channel independently and computed our limits by restricting ourselves to the channel yielding the strongest exclusion. In this way, we have omitted all correlations that could improve the bounds. Finally, in some of the considered searches, the background estimation in the various signal regions depends on an extrapolation from designated control regions. Consequently, the possibility of control region contamination by signal events should also be taken into account. This contamination, however, depends on the signal model being explored, and the information on how we should quantify it is not public. This has therefore not been pursued in our work.

The combined effect of all these features leads to results that are compatible with the experimental ones within $10 \%-20 \%$ [42]. We stress however that the relative uncertainty is much smaller. Many of the errors arising from our simplified recast should lead to an overall mis-estimation of a given bound. Yet we do not expect these recast errors to be sensitive to the size of the flavor mixing. In other words, when taking ratios of bounds (which we effectively do when considering how bounds change), this systematic uncertainty should largely drop out.

We have verified the consistency of our methodology for the analyses under consideration, and validated in this way our reimplementation procedure. More details are given in the rest of this section, in appendix $\mathrm{A}$ as well as in ref. [42].

\subsection{ATLAS: multijets $+\not_{T}+$ lepton veto}

The ATLAS-SUSY-2013-04 search [39] is a supersymmetry-oriented search which focuses on a multijet signature accompanied by large missing transverse energy and no isolated hard leptons (electron or muon). In the context of our model description of section 2, it targets the production of gluino pairs, squark pairs or gluino-squark associated pairs that subsequently decay into missing transverse energy and jets. The selection strategy relies on dedicated multijet triggers with a minimal requirement of five (six) very energetic central jets with a transverse energy $E_{T}>55 \mathrm{GeV}\left(E_{T}>45 \mathrm{GeV}\right)$ and a pseudorapidity satisfying $|\eta|<3.2$. Events are then collected into two types of signal regions, the so-called 'multijet + flavor stream' and 'multijet $+M_{J}^{\Sigma}$ stream' categories, which yields a total of 19 overlapping signal regions.

In the 'multijet + flavor stream' signal region category, the events are classified according to requirements on the number of jets exhibiting specific properties. In a first set of regions, the events are required to feature exactly $8(8 \mathrm{j} 50), 9(9 \mathrm{j} 50)$ or at least $10(\geq 10 \mathrm{j} 50)$ jets with a transverse momentum $p_{T}>50 \mathrm{GeV}$ and a pseudorapidity $|\eta|<2$. In a second set of regions, they are constrained to contain exactly $7(7 \mathrm{j} 80)$ or at least $8(\geq 8 \mathrm{j} 80)$ jets with a transverse momentum $p_{T}>80 \mathrm{GeV}$ and a pseudorapidity $|\eta|<2$. Except for the $\geq 10 \mathrm{j} 50$ region, a further subdivision is made according to the number of $b$-tagged jets $(0$, 1 or at least 2) with a pseudorapidity $|\eta|<2.5$ and a transverse momentum $p_{T}>40 \mathrm{GeV}$. The signal selection strategy finally relies on the missing transverse energy significance, 
$\mathbb{E}_{T} / \sqrt{H_{T}}>4 \mathrm{GeV}^{1 / 2}$, where $H_{T}$ is defined as the sum of the hadronic transverse energy of all jets with $E_{T}$ larger than $50 \mathrm{GeV}$. For SM processes, this variable is expected to be small, and almost insensitive to the jet multiplicity. A further background reduction is thus obtained by requiring the same, relatively high missing transverse-energy significance in all signal regions.

The 'multijet $+M_{J}^{\Sigma}$ stream' signal region category relies on an extra variable, $M_{J}^{\Sigma}$, that is defined as the invariant mass obtained after combining the momenta of all fat jets (whose radius is $R=1$ ) with a transverse momentum larger than $100 \mathrm{GeV}$ and a pseudorapidity smaller than 1.5 in absolute value. Unfortunately, the MADANALYsis 5 framework is currently unable to handle fat jets. Consequently, we refrain ourselves from implementing this type of signal region flow in our recasting procedure.

This work features the first use of a reimplementation of the ATLAS-SUSY-2013-04 search in the MADANALYsis 5 framework. Details on its validation are therefore given in appendix A.

\subsection{CMS: single lepton + at least four jets (including at least one $b$-jet) $+\boldsymbol{E}_{T}$}

The CMS-SUS-13-011 search [38] is a stop search that targets stop pair-production and two possible decay modes of the stop, $\tilde{t} \rightarrow t \tilde{\chi}^{0} \rightarrow W^{+} b \tilde{\chi}^{0}$ and $\tilde{t} \rightarrow b \tilde{\chi}^{+} \rightarrow b W^{+} \tilde{\chi}^{0}$, that lead to similar final-state topologies. In the model description of section 2 we have assumed that the charginos are decoupled, which is a fair assumption if the $\mu$-term is large. Nevertheless, we still include this search in our analysis as its signal regions cover signatures which are related to the top-neutralino decay of the stop. The CMS-SUS-13-011 search targets events where one of the $W$-bosons decays hadronically, while the other one decays leptonically, into an electron or a muon (the $\tau$ channel is ignored). It contains two analysis flows, a first one using a predefined selection strategy and that is hence 'cut-based', and a second one relying on a boosted decision tree (BDT) technique. Although the BDT analysis provides a sensitivity that is $40 \%$ better, the absence of related public information prevents the community from making use of it for phenomenological purposes. We therefore focus only on the cut-based analysis strategy.

The object definition and event preselection criteria require the presence of a single isolated lepton with a transverse momentum $p_{T}>30 \mathrm{GeV}(25 \mathrm{GeV})$ and a pseudorapidity $|\eta|<1.4$ (2.1) in the case of an electron (a muon). Moreover, no jet can be found in a cone of $R=0.4$ centered on the lepton. A veto is further enforced on events featuring an additional (loosely) isolated lepton or a track with an electric charge opposite to the one of the primary lepton, as well as on events containing hadronic taus. Furthermore, at least four jets with a transverse momentum $p_{T}>30 \mathrm{GeV}$ and a pseudorapidity $|\eta|<2.4$ are required, with at least one of them being $b$-tagged. The preselection finally imposes that $\mathbb{E}_{T}>100 \mathrm{GeV}$, that the azimuthal angle between the missing momentum and the first two leading jets is above 0.8 and that the transverse mass constructed from the lepton and the missing momentum is larger than $120 \mathrm{GeV}$.

Various signal regions are then defined from several considerations. First, one designs categories dedicated to probe each of the two considered stop decay modes, $\tilde{t} \rightarrow t \tilde{\chi}^{0}$ and $\tilde{t} \rightarrow b \tilde{\chi}^{+}$. To this aim, one imposes constraints on the hadronic top reconstruction quality 
in the case of the region category related to the top-neutralino stop decay as well as on the amount of missing energy. In the case of a stop into a neutralino decay, four overlapping signal regions are defined after requiring $\mathbb{E}_{T}$ to be larger than $150,200,250$ and $300 \mathrm{GeV}$, respectively. In the case of a stop into chargino decay, four regions are again defined, but using missing energy thresholds of 100, 150, 200 and $250 \mathrm{GeV}$. Next, the categories are further subdivided into regions whose goal is to probe large or small mass differences $\Delta M$ between the stop and the LSP. Large $\Delta M$ regions are defined by enforcing the transverse variable $M_{T 2}^{W}$ [62] to be larger than $200 \mathrm{GeV}$ and the leading $b$-jet to have a $p_{T}$ greater than $100 \mathrm{GeV}$, this last criterion being only relevant for the case of a stop decay into a chargino.

Information on the implementation and validation of this analysis in the MADANALYSIS 5 framework can be found in ref. [42] and on INSPIRE [63].

\subsection{CMS: at least 3 jets $+\mathbb{E}_{T}+$ lepton veto}

The CMS-SUS-13-012 analysis [40] is a search for supersymmetry, which focuses on the pair-production of gluinos and squarks. The main final state sought in this search is comprised of a multijet system and missing transverse energy, without any isolated leptons. It is hence directly sensitive to our simplified models in which such signatures would be copiously produced.

The event selection requires at least three jets with a transverse momentum $p_{T}>50 \mathrm{GeV}$ and a pseudorapidity satisfying $|\eta|<2.5$. The total hadronic activity of the events is then estimated by means of the $H_{T}$ variable defined as the scalar sum of the transverse momenta of all jets satisfying the above requirements. The amount of missing transverse energy in the events is computed via the $\vec{H}_{T}$ vector obtained by a vector sum of the transverse momenta of all jets with $p_{T}>30 \mathrm{GeV}$ and a pseudorapidity smaller than 5 in absolute value. The analysis requires that $H_{T}>500 \mathrm{GeV}, H_{T}>200 \mathrm{GeV}$ (where $\left.H_{T}=\left|\vec{H}_{T}\right|\right)$ and events in which one of the three hardest jets is aligned with $\vec{H}_{T}$ are vetoed by requiring $\left|\Delta \phi\left(p_{T}^{j}, \vec{H}_{T}\right)\right|>0.5$ for the two hardest jets, and $\left|\Delta \phi\left(p_{T}^{j}, \vec{H}_{T}\right)\right|>0.3$ for the third hardest one. The selection strategy finally includes a veto on any isolated lepton whose transverse momentum is larger than $10 \mathrm{GeV}$. The events that pass these selection criteria are then categorized into 36 non-overlapping signal regions defined by the number of jets and the value of the $H_{T}$ and $H_{T}$ variables.

Information on the implementation and validation of this analysis in the MADANALYSIS 5 framework can be found in ref. [42] and on INSPIRE [64].

\subsection{ATLAS: scharm pair-production using charm-tagging + lepton veto}

The ATLAS-SUSY-2014-03 search [41] is a supersymmetry search that looks for scharm pair production followed by the $\tilde{c} \rightarrow c \tilde{\chi}^{0}$ decay. The final state is thus comprised of two charm-jets and missing energy. The experimental analysis therefore targets events that are required to present a large amount of missing transverse energy, $\mathbb{E}_{T}>150 \mathrm{GeV}$, at least two very hard jets with transverse momenta greater than 130 and $100 \mathrm{GeV}$, respectively, and no isolated leptons. The two jets are then demanded to be $c$-tagged. This constitutes the main novel feature of this search, that involves dedicated charm-tagging techniques 
based on algorithms optimized with neural networks. Additional requirements are finally applied by constraining the so-called contransverse mass [65].

Mimicking charm-tagging algorithms is beyond the ability of our simplified detector emulation that relies on DELPHES. We therefore recast this search following a different strategy, not based on the MADANALYsis 5 framework. A very conservative (over)-estimate of the bounds is instead derived from the cross-section limits presented in the experimental publication [41] that we compare to theoretical predictions for the production cross section of a system made of two charm quarks and two neutralinos that originate from the decay of two superpartners. More precisely, the theoretical cross section is calculated from the sum of the production cross sections $\sigma_{x y}$ of any pair of superpartners $x$ and $y$, the individual channels being reweighted by the corresponding branching ratios (BR) so that a final state made of two charm quarks and two neutralinos is ensured,

$$
\begin{aligned}
& \sigma^{\mathrm{SUSY}}\left(2 c+\mathbb{E}_{T}\right)=\sum_{i, j=1,2}\left[\left(\frac{\sigma_{\tilde{u}_{i} \tilde{u}_{j}^{*}}+\sigma_{\tilde{u}_{j} \tilde{u}_{i}^{*}}}{1+\delta_{i j}}+\sigma_{\tilde{u}_{i} \tilde{u}_{j}}+\sigma_{\tilde{u}_{i}^{*} \tilde{u}_{j}^{*}}\right) \times \mathrm{BR}_{\tilde{u}_{i} \rightarrow c \tilde{\chi}_{1}^{0}} \times \mathrm{BR}_{\tilde{u}_{j} \rightarrow c \tilde{\chi}_{1}^{0}}\right] \\
& +\sigma_{\tilde{g} \tilde{\chi}_{1}^{0}} \times \sum_{i=1,2}\left[\mathrm{BR}_{\tilde{g} \rightarrow c \tilde{u}_{i}} \times \mathrm{BR}_{\tilde{u}_{i} \rightarrow c \tilde{\chi}_{1}^{0}}\right] \\
& +\sum_{i, j=1,2}\left(\sigma_{\tilde{g} \tilde{u}_{j}}+\sigma_{\tilde{g} \tilde{u}_{j}^{*}}\right) \times\left[\mathrm{BR}_{\tilde{g} \rightarrow c \tilde{u}_{i}} \times \mathrm{BR}_{\tilde{u}_{i} \rightarrow c \tilde{\chi}_{1}^{0}}\right. \\
& \left.\quad+\left(\mathrm{BR}_{\tilde{g} \rightarrow c \tilde{u}_{i}} \times \mathrm{BR}_{\tilde{u}_{i} \rightarrow t \tilde{\chi}_{1}^{0}}+\mathrm{BR}_{\tilde{g} \rightarrow t \tilde{u}_{i}} \times \mathrm{BR}_{\tilde{u}_{i} \rightarrow c \tilde{\chi}_{1}^{0}}\right) \times \mathrm{BR}_{\tilde{u}_{j} \rightarrow c \tilde{\chi}_{1}^{0}}\right] \\
& +\sigma_{\tilde{g} \tilde{g}} \times \sum_{i, j=1,2}\left[\mathrm{BR}_{\tilde{g} \rightarrow c \tilde{u}_{i}} \times \mathrm{BR}_{\tilde{g} \rightarrow c \tilde{u}_{j}}+\mathrm{BR}_{\tilde{g} \rightarrow t \tilde{u}_{i}} \times \mathrm{BR}_{\tilde{u}_{i} \rightarrow c \tilde{\chi}_{1}^{0}} \times \mathrm{BR}_{\tilde{g} \rightarrow t \tilde{u}_{j}} \times \mathrm{BR}_{\tilde{u}_{j} \rightarrow c \tilde{\chi}_{1}^{0}}\right. \\
& \left.\quad+2 \mathrm{BR}_{\tilde{g} \rightarrow t \tilde{u}_{i}} \times \mathrm{BR}_{\tilde{g} \rightarrow c \tilde{u}_{j}}\left(\mathrm{BR}_{\tilde{u}_{i} \rightarrow c \tilde{\chi}_{1}^{0}}+\mathrm{BR}_{\tilde{u}_{i} \rightarrow t \tilde{\chi}_{1}^{0}} \times \mathrm{BR}_{\tilde{u}_{j} \rightarrow c \tilde{\chi}_{1}^{0}}\right)\right] .
\end{aligned}
$$

We then implicitly (and incorrectly) assume that all the other requirements of the ATLAS analysis described above are fulfilled. In particular, the fact that the neutralino is almost massless in our parameterization enforces a large amount of missing energy.

\section{Results}

In this section, we present and discuss the main results of our reinterpretation study. As a preliminary, we introduce two concepts that we call 'signal migration' and 'signal depletion'. Each of the recasted experimental analyses targets several event topologies which are assigned to one (exclusive) or more (inclusive) signal regions. These signal regions then serve as exclusion channels for the new physics scenarios that are probed. In our case, the typical effect of the flavor mixing and the squark mass splitting will be to modify the branching fractions of the particles, and perhaps also the rate of some production processes. Consequently, signal regions that are usually largely populated by signal events in the case where there is no squark mixing can turn out to be depleted from events once flavor violation in the squark sector is allowed, and conversely, signal regions that are not sensitive to any supersymmetric signal in the flavor-conserving case can become populated. One thus expects a migration of the signal across the different regions with squark flavor violation. 
As a concrete example, we compare the event topologies resulting from the decay $\tilde{t} \rightarrow q \tilde{\chi}^{0}$ in a flavor-conserving model to the case of a model with stop-scharm mixing. If the stop decays solely into tops, one expects signatures which would include a $b$-tagged jet, in addition to either two other jets or a lepton-neutrino pair that originates from the decay of the $W$-boson. Alternatively, if the stop can also decay into charm-jets, the jet multiplicity distribution of the stop decay products peaks to a smaller value. Furthermore, a decay into a charm jet, rather than into a top quark, is not bounded by the top mass and can result in larger neutralino energies which manifest as a signature with a larger amount of missing energy.

The direct interpretation of this example in the context of the searches under consideration is simple. In comparing a flavor-conserving to a flavor-violating scenario, one expects that signal regions which are defined according to requirements on the missing energy, the number of isolated leptons, the number of jets and the number of $b$-tagged jets, to redistribute signal events between one another. This migration of signal events could cause a signal region depletion or population.

In the rest of this section, we present and discuss our results in the $m_{\tilde{u}}-m_{\tilde{g}}$ plane for the model parameterization described in section 2 .

\subsection{ATLAS: multijets $+\mathbb{E}_{T}+$ lepton veto}

The ATLAS-SUSY-2013-04 search [39] has been designed to target supersymmetric signals with large hadronic activity in conjunction with missing energy. The requirement for a minimum of seven hard jets implies that this search should be most sensitive to $\tilde{g} \tilde{g}$ and $\tilde{g} \tilde{q}$ production with subsequent decays into top quarks. In this subsection we discuss how the exclusion limits shown in the $\left(m_{\tilde{u}}, m_{\tilde{g}}\right)$ plane depend on the two flavor-violating parameters: the squark mass splitting $\Delta m$, and the squark mixing angle $\theta_{23}^{R}$. We take as benchmark the case of a light stop and a decoupled scharm that are not mixed, with $\Delta m=-500 \mathrm{GeV}$ and $\sin \theta_{23}^{R}=0$. In the upper panel of figure 1 we show the reach of this ATLAS search for this scenario. In the other sub-figures of figure 1, we collect a representative set of results with various $\Delta m$ and $\theta_{23}^{R}$ values, which depict the interesting changes in the analysis reach due to flavor effects.

For cases in which the lightest squark is stop-like (scenarios of class S.II with $\Delta m<0$ ), it is evident that the sensitivity of this search to gluino masses of about $1.4 \mathrm{TeV}$ is reduced by a non-zero stop-scharm mixing. Due to flavor mixing, final states with charm quarks instead of top quarks can arise, changing in this way the possible event topology and depleting some of the multijet signal regions that require a large jet multiplicity. A similar effect happens to the constraint on the mass of the lightest squark of about $500 \mathrm{GeV}$ when the gluino is heavy, with a mass ranging up to $2 \mathrm{TeV}$. This constraint originates mostly from direct squark-gluino associated production, and the global jet multiplicity of the event is again reduced when decays into charm quarks are allowed.

Interestingly, close to the degeneracy line $m_{\tilde{q}}=m_{\tilde{g}}$, the opposite effect occurs and the sensitivity increases when the mixing is turned on. For $\sin \theta_{23}^{R}=0$, the two-body decay $\tilde{g} \rightarrow \tilde{q} t$ is kinematically forbidden when $m_{\tilde{g}}-m_{\tilde{t}}<m_{t}$ so that the dominant gluino decay mode proceeds through a three-body channel, $\tilde{g} \rightarrow t \bar{t} \tilde{\chi}_{1}^{0}$. When the stop and the scharm 

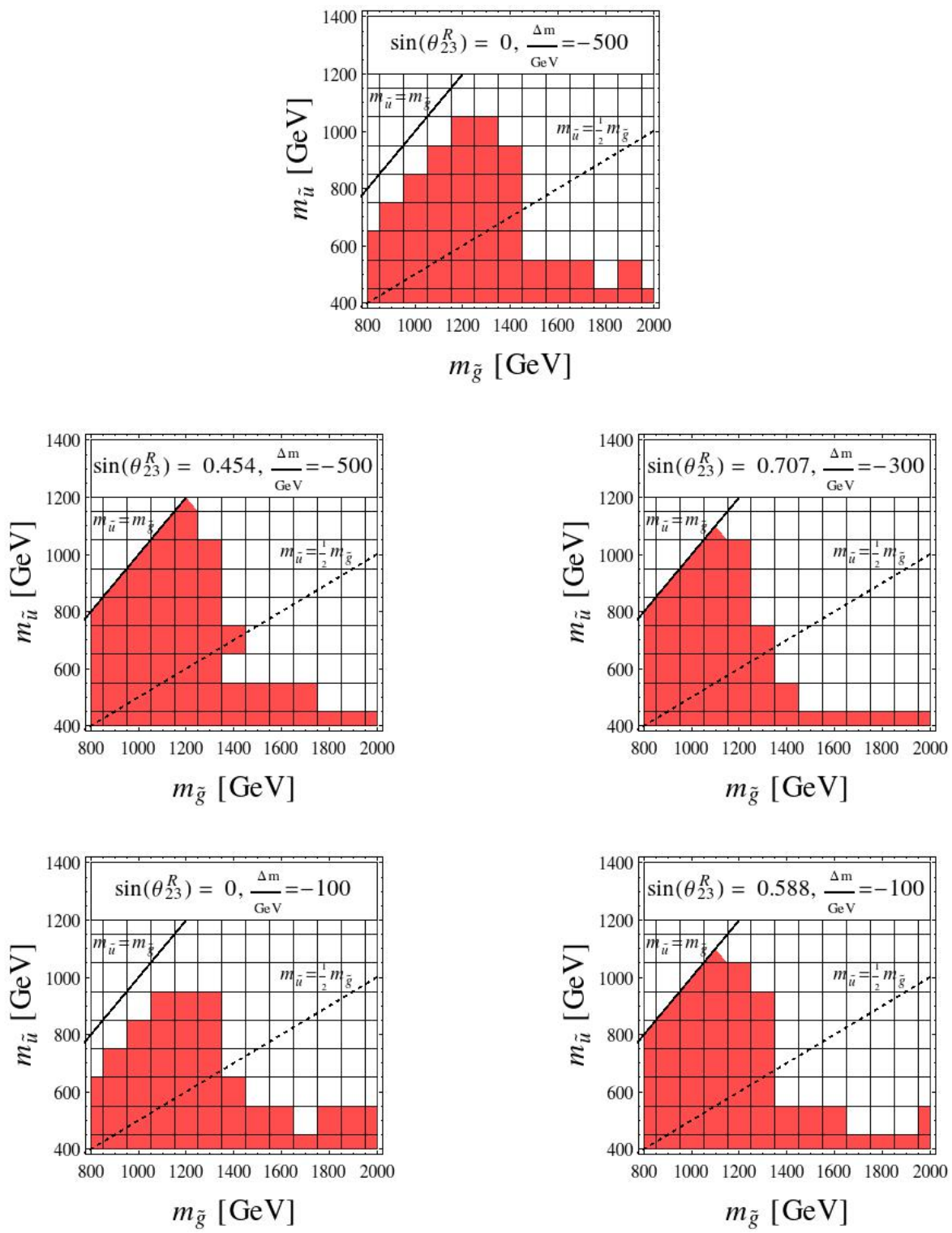

Figure 1. Sensitivity of the ATLAS multijet $+\mathbb{E}_{T}+$ lepton veto search of ref. [39] for different values of the $\Delta m$ and $\theta_{23}^{R}$ parameters (the exact values being indicated in the top bar of each subfigure). The excluded regions are shown in red in the $\left(m_{\tilde{u}}, m_{\tilde{g}}\right)$ plane, where $m_{\tilde{u}}$ is the mass of the lightest squark (a stop-like squark here since $\Delta m<0$ ). The upper panel describes the reference scenario in which the lightest squark is a pure stop state and the scharm is almost decoupled.

mix, the two-body decay mode $\tilde{g} \rightarrow \tilde{q} c$ is open and dominates, even for relatively small mixing angles. In this case, the top quarks from the subsequent squark decay are more energetic than in the three-body decay case. The decay products of these tops are therefore experimentally easier to detect so that the search sensitivity is enhanced.

In figure 2, we focus on scenarios of type S.I where the lightest squark is scharm-like and $\Delta m>0$. In the upper panel of the figure, we consider a reference scenario in which the stop is decoupled and both squarks do not mix. In comparison with the S.II benchmark 

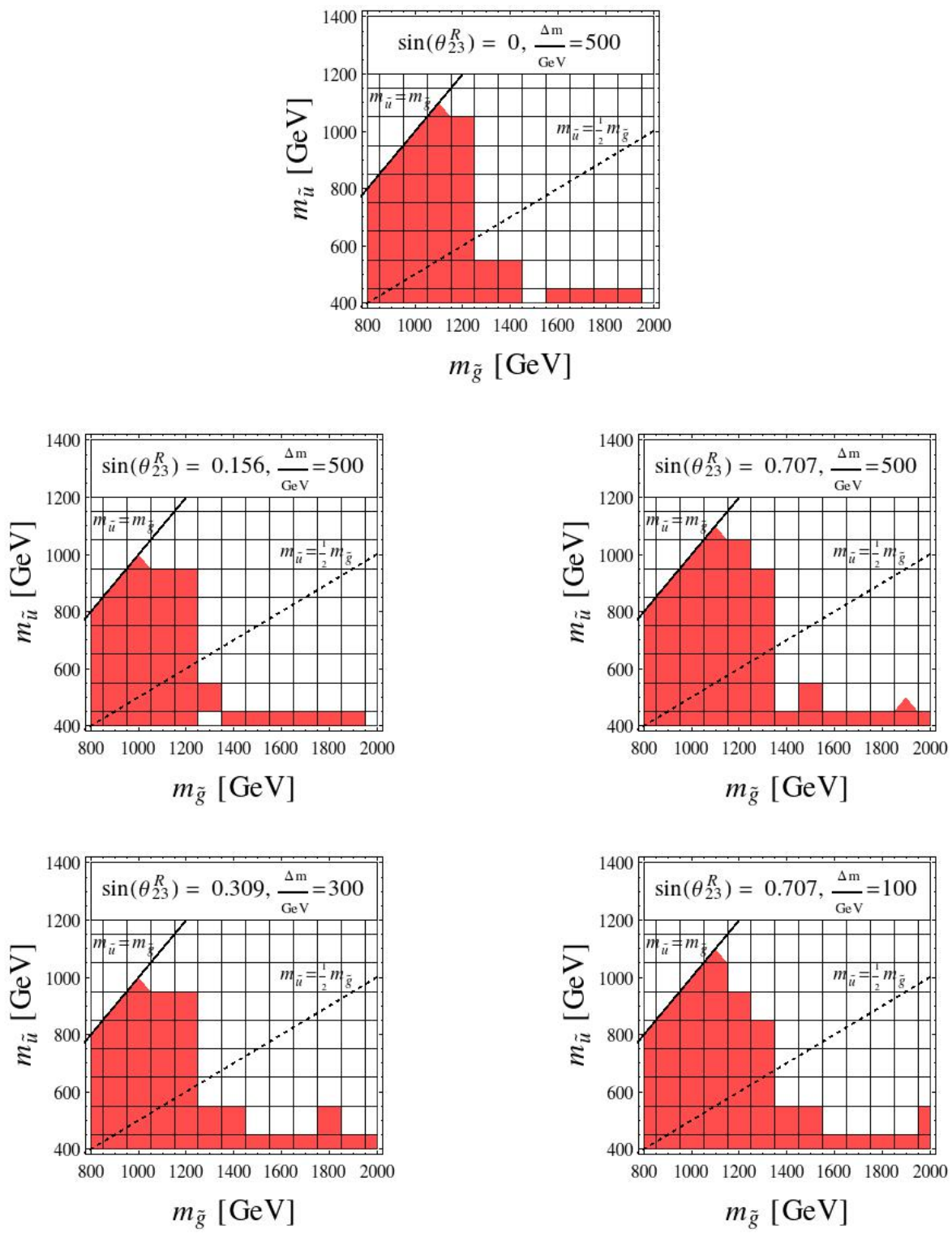

Figure 2. Sensitivity of the ATLAS multijet $+\mathbb{E}_{T}+$ lepton veto search of ref. [39] for different values of the $\Delta m$ and $\theta_{23}^{R}$ parameters (the exact values being indicated in the top bar of each subfigure). The excluded regions are shown in red in the $\left(m_{\tilde{u}}, m_{\tilde{g}}\right)$ plane, where $m_{\tilde{u}}$ is the mass of the lightest squark (a scharm-like squark here since $\Delta m>0$ ). The upper panel describes the reference scenario in which the lightest squark is a pure scharm state and the stop is almost decoupled.

scenario, the search has a more limited reach. The reason is twofold. On the one hand, the lighter scharm-like state is produced more copiously than the the stop-like one via $\tilde{g} \tilde{q}$ production, and on the other hand, the gluino branching fraction to charm-jets is increased, leading to a depletion of the signal regions with a large jet multiplicity. A non-vanishing stop-scharm mixing allows the gluino and the scharm-like state to decay into top quarks, thus increasing the number of jets in the event and repopulating the search signal regions 
by signal migration. The impact of such a change is, however, rather modest, and becomes significant only for large mixings. This result is due to the small branching fractions of the gluino and the scharm-like state into top quarks which are suppressed by the top mass, and could therefore become substantial only for large values of the mixing angle.

\subsection{CMS: single lepton + at least four jets (including at least one $b$-jet) $+\mathbb{E}_{T}$}

The CMS-SUS-13-011 search of ref. [38] has been designed to look for the semileptonic decay of a stop pair. Consequently, we expect this search to be rather insensitive to spectra where the lightest squark is scharm-like (scenarios of type S.I). The results in figure 3 and figure 4 show that this is indeed the case with the exception of scenarios in which $0<\Delta m<200 \mathrm{GeV}$ and/or in which the mixing angle $\theta_{23}^{R}$ is large. In these cases, constraints on the gluino masses of about $1 \mathrm{TeV}$ find their origin in gluino-squark associated production followed by their decay to two neutralinos and three quarks, at least two of which are tops. In all the other cases with a light scharm-like squark, the top mass severely suppresses any branching fraction to a final state containing a top quark and therefore reduces the sensitivity of the search.

For a stop-like lightest squark (scenarios of class S.II), the reach of this search is much more significant. In addition to gluino pair-production, direct squark pair-production can also yield a significant number of top-pairs. As a result, light squark masses around $500 \mathrm{GeV}$ are excluded independently of the gluino mass, unless the mixing angle is very large. In the latter case (right column of figure 3), the signal regions are depleted when the squarks can decay not only to top quarks, but also to charm-jets which have the advantage of a larger phase-space. Similarly to the ATLAS multijet search, the sensitivity to nearly degenerate squark and gluino states increases with a non-zero stop-scharm mixing angle.

\subsection{CMS: at least 3 jets $+\boldsymbol{E}_{T}+$ lepton veto}

Next, we consider the CMS-SUS-13-012 search of ref. [40] that is complementary to the previous CMS search. This search targets the purely hadronic decays of pair-produced superpartners, and therefore vetoes final-states which include leptons. The dependence of the sensitivity on the squark mass splitting and the mixing angle is depicted in figure 5 and figure 6. Taking as a reference the non-mixing scenario of class S.II with a decoupled charm-squark (upper panel of figure 5), we observe that the sensitivity is enhanced in all the other cases. This is a direct consequence of the relatively small jet-multiplicity requirement of this CMS analysis alongside the lepton veto. Those imply that events featuring charmjets which come from $\tilde{g} \tilde{g}$ and $\tilde{g} \tilde{q}$ production (whose respective cross-sections are large and mildly depend on the squark mass) are more likely to pass all the selection steps than those featuring top quarks. The latter have indeed a non-negligible branching fraction into leptons, and the top mass tends to limit the amount of missing energy in the events (the neutralino $\left.p_{T}\right)$. Consequently, one expects that for the case in which the lightest squark is stop-like, a non zero mixing will increase the reach of the search, which is indeed the case as seen through figure 5 .

In an analogous way, one can explain the pattern of exclusion limits for scenarios of class S.I with a scharm-like lightest squark illustrated in figure 6 . The gluino mass bound 

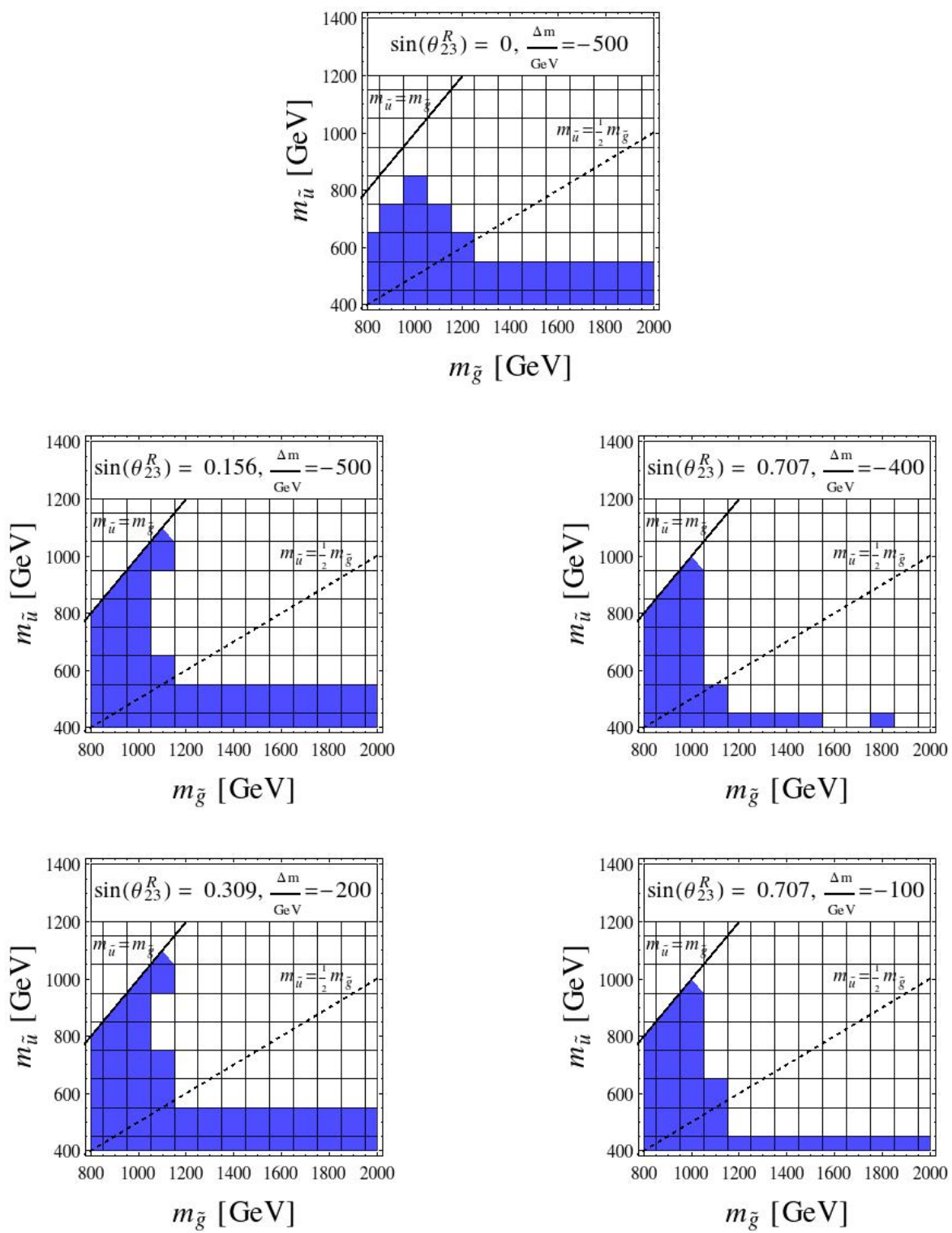

Figure 3. Sensitivity of the CMS stop search in the single lepton mode of ref. [38] for different values of the $\Delta m$ and $\theta_{23}^{R}$ parameters (the exact values being indicated in the top bar of each subfigure). The excluded regions are shown in blue in the $\left(m_{\tilde{u}}, m_{\tilde{g}}\right)$ plane, where $m_{\tilde{u}}$ is the mass of the lightest squark (a stop-like squark here since $\Delta m<0$ ). The upper panel describes the reference scenario in which the lightest squark is a pure stop state and the scharm is almost decoupled.

in the reference scenario is somewhat stronger here than in the stop-like case, but it is reduced for increasing mixing angles.

\subsection{ATLAS: scharm pair-production using charm-tagging + lepton veto}

The ATLAS-SUSY-2014-03 charm-squark search of ref. [41] targets the production of a pair of charm squarks via a signature comprised of two charm-tagged jets and missing transverse energy $\mathbb{E}_{T}$. We recall that our implementation of this search is very conservative and is 

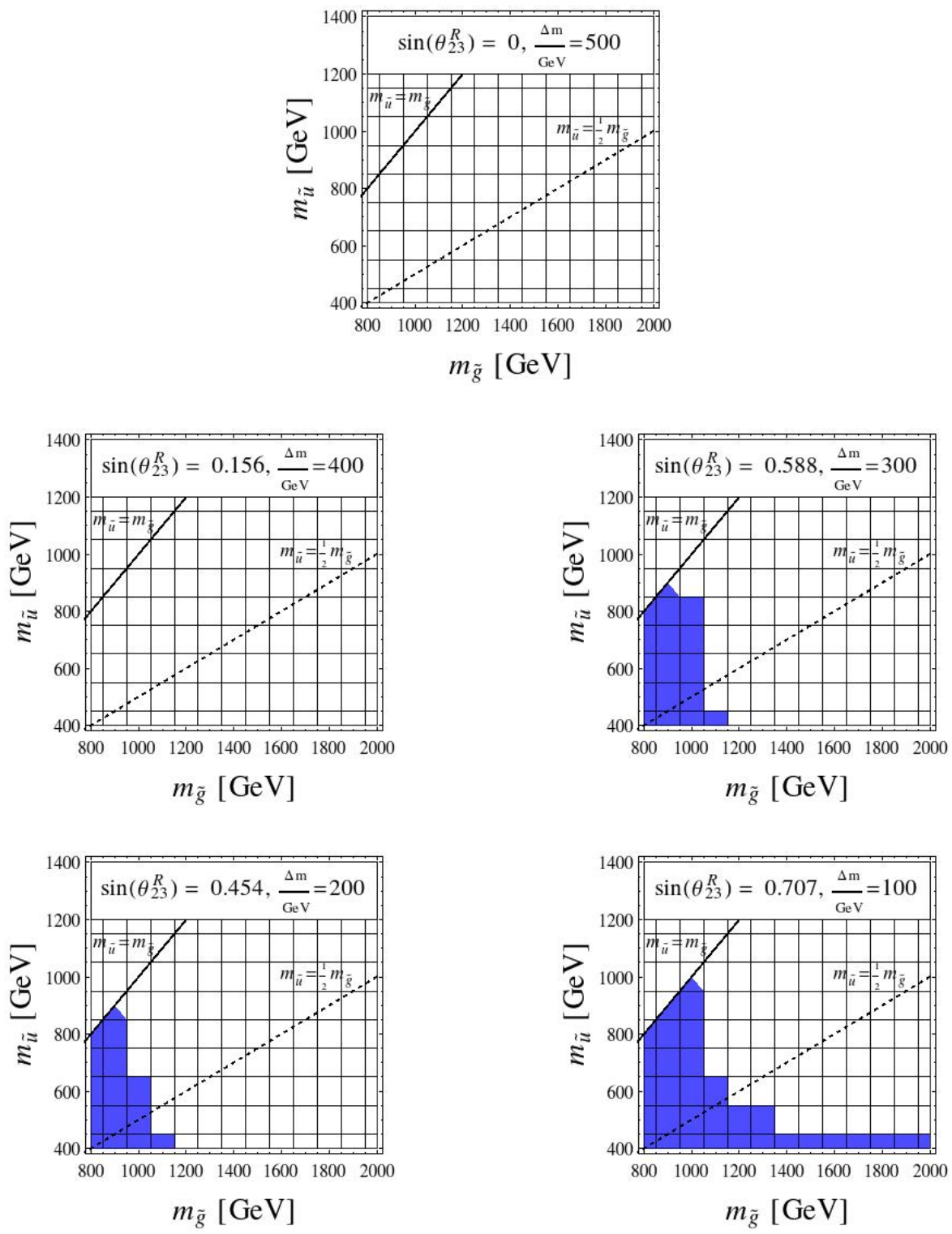

Figure 4. Sensitivity of the CMS stop search in the single lepton mode of ref. [38] for different values of the $\Delta m$ and $\theta_{23}^{R}$ parameters (the exact values being indicated in the top bar of each subfigure). The excluded regions are shown in blue in the $\left(m_{\tilde{u}}, m_{\tilde{g}}\right)$ plane, where $m_{\tilde{u}}$ is the mass of the lightest squark (a scharm-like squark here since $\Delta m>0$ ). The upper panel describes the reference scenario in which the lightest squark is a pure scharm state and the stop is almost decoupled.

only based on cross sections and branching ratios. The sensitivity of this search to the model studied in this work is presented in figure 7 and figure 8 .

In case where the lightest squark is scharm-like (scenarios of class S.I), the search is sensitive to two specific regions of the parameter-space. The first, with lower squark masses of about $500 \mathrm{GeV}$, is independent of the gluino mass and extends up to $m_{\tilde{g}} \sim 2 \mathrm{TeV}$. In this domain, squark pair production is sufficient to yield an exclusion of the model regardless of whether gluinos can be produced. In contrast, in the second domain, the squarks are 

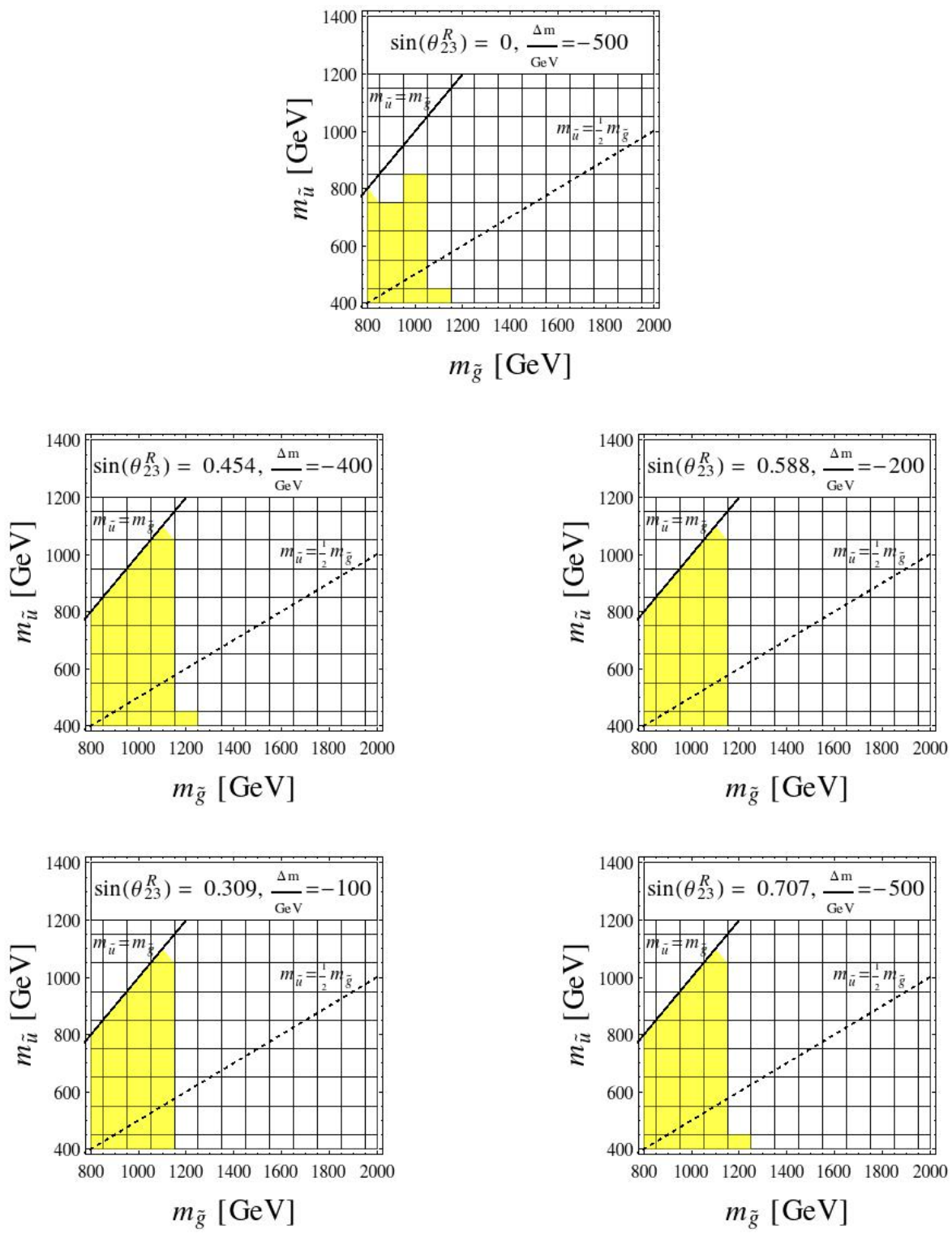

Figure 5. Sensitivity of the CMS supersymmetry search in the multijet plus missing energy channel of ref. [40] for different values of the $\Delta m$ and $\theta_{23}^{R}$ parameters (the exact values being indicated in the top bar of each subfigure). The excluded regions are shown in yellow in the $\left(m_{\tilde{u}}, m_{\tilde{g}}\right)$ plane, where $m_{\tilde{u}}$ is the mass of the lightest squark (a stop-like squark here since $\Delta m<0$ ). The upper panel describes the reference scenario in which the lightest squark is a pure stop state and the scharm is almost decoupled.

heavier, so that an exclusion of the model must rely on the production of gluinos followed by their subsequent decays into charm-jets. In this case, the reach depends on the gluino mass that limits the production cross section. This feature also explains the effect of the different mass splittings and mixings. For large enough positive mass splitting, $\Delta m>200 \mathrm{GeV}$, there is a light scharm-like state whose mixing dependent branching fractions to charm-jets leads to an exclusion roughly independent of the gluino mass. Although the exact value 

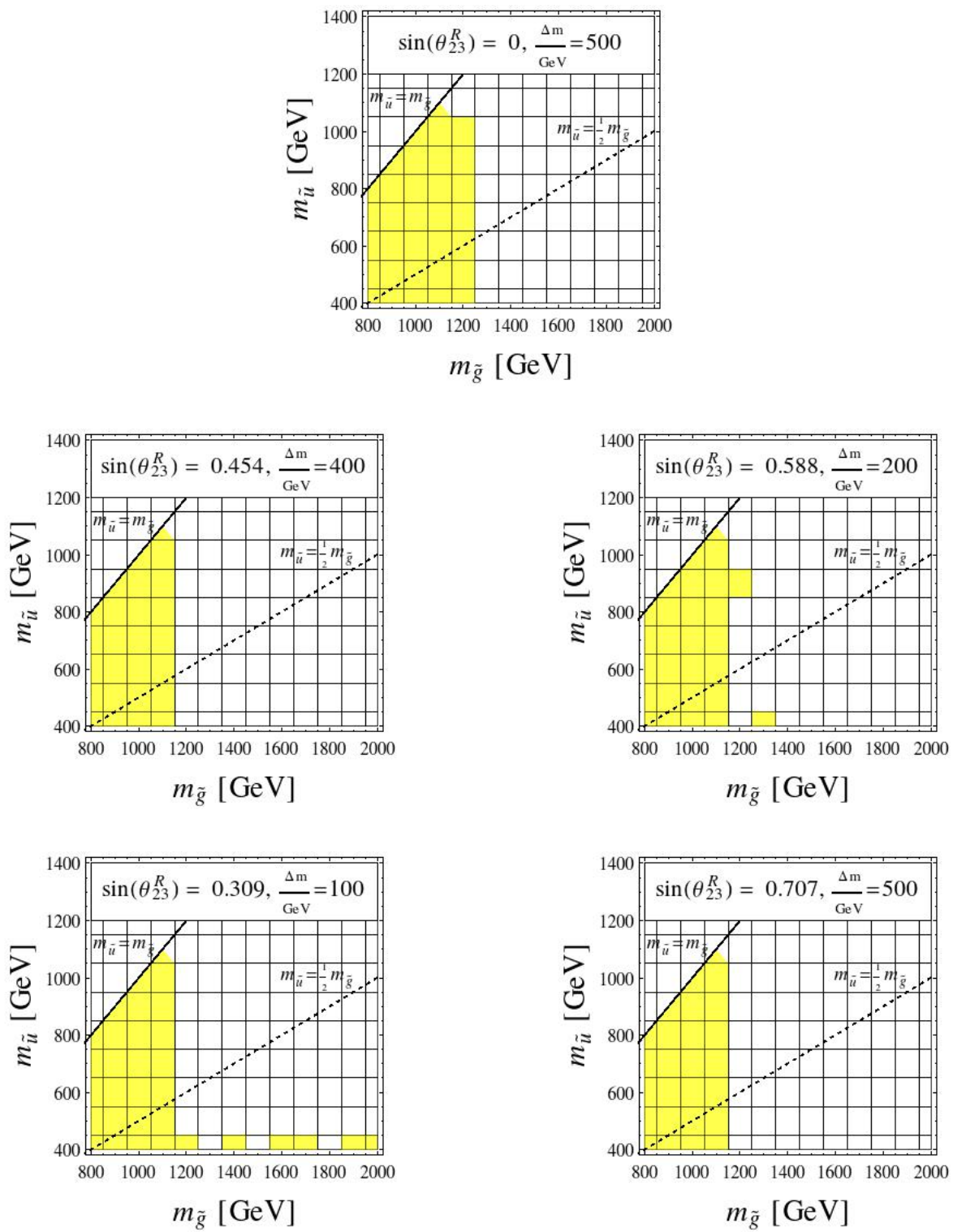

Figure 6. Sensitivity of the CMS supersymmetry search in the multijet plus missing energy channel of ref. [40] for different values of the $\Delta m$ and $\theta_{23}^{R}$ parameters (the exact values being indicated in the top bar of each subfigure). The excluded regions are shown in yellow in the $\left(m_{\tilde{u}}, m_{\tilde{g}}\right)$ plane, where $m_{\tilde{u}}$ is the mass of the lightest squark (a scharm-like squark here since $\Delta m>0$ ). The upper panel describes the reference scenario in which the lightest squark is a pure scharm state and the stop is almost decoupled.

of the excluded mass limit changes with the mixing angle, the general shape persists. For smaller values of the squark mass splitting, both squark states become accessible at the LHC, and with sufficiently large mixing, they contribute significantly to the production of events with several charm-jets.

The search loses sensitivity if the lightest squark is a pure stop state (scenarios of class S.II). Consequently, the exclusion reach for negative mass splittings strongly relies on the 

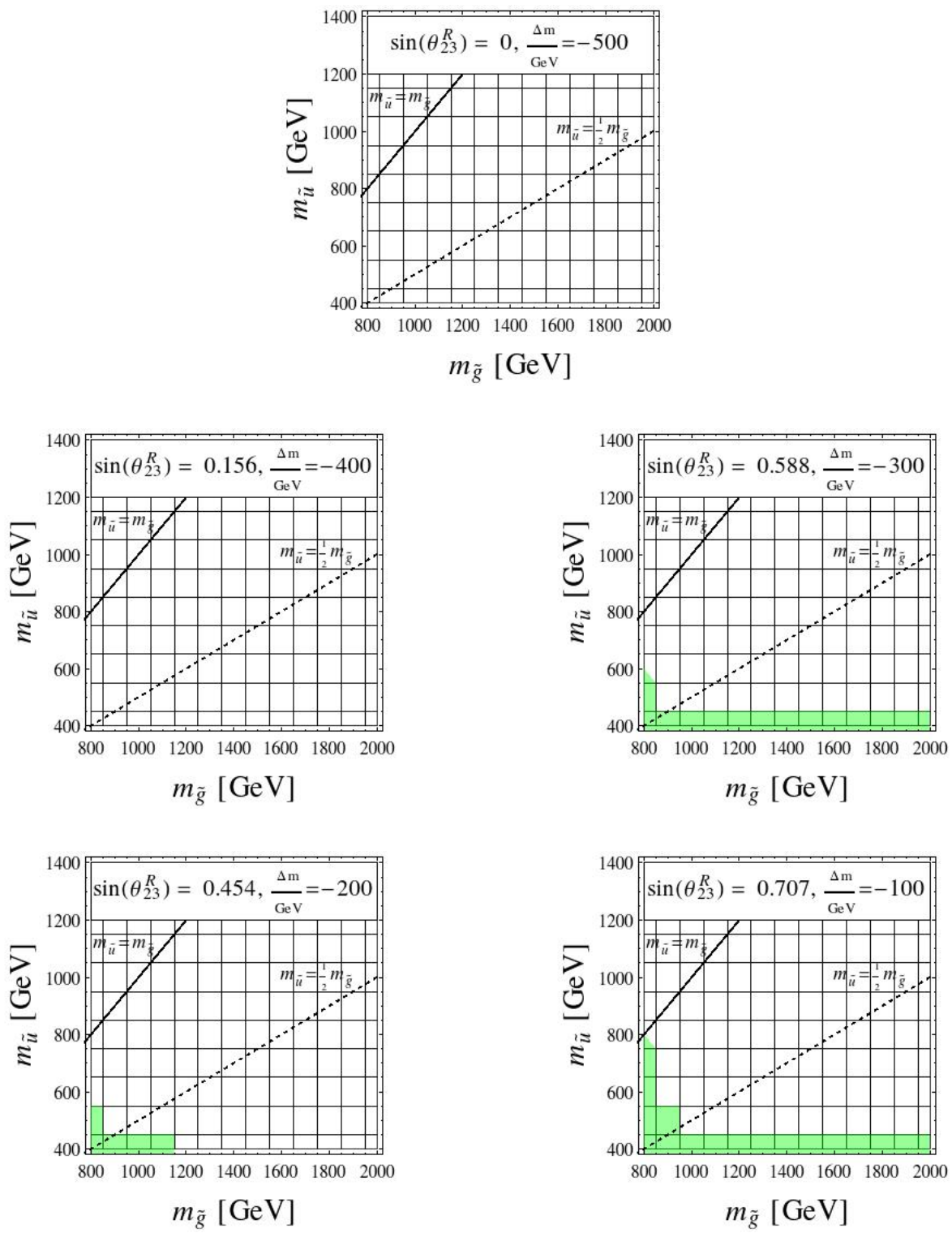

Figure 7. Sensitivity of the ATLAS charm-squark search of ref. [41] for different values of the $\Delta m$ and $\theta_{23}^{R}$ parameters (the exact values being indicated in the top bar of each subfigure). The excluded regions are shown in green in the $\left(m_{\tilde{u}}, m_{\tilde{g}}\right)$ plane, where $m_{\tilde{u}}$ is the mass of the lightest squark (a stop-like squark here since $\Delta m<0$ ). The upper panel describes the reference scenario in which the lightest squark is a pure stop state and the scharm is almost decoupled.

value of the mixing angle. A large mixing indeed opens the possible supersymmetric decays into charm-jets. Alternatively, lowering the mass splitting also improves the reach as it makes the heavier state, that is scharm-like, accessible.

\subsection{Combined reach}

It is interesting to discuss the combined reach of the four previously pursued searches for a few benchmarks points. To this end, we overlay all four exclusion contours on top of each 

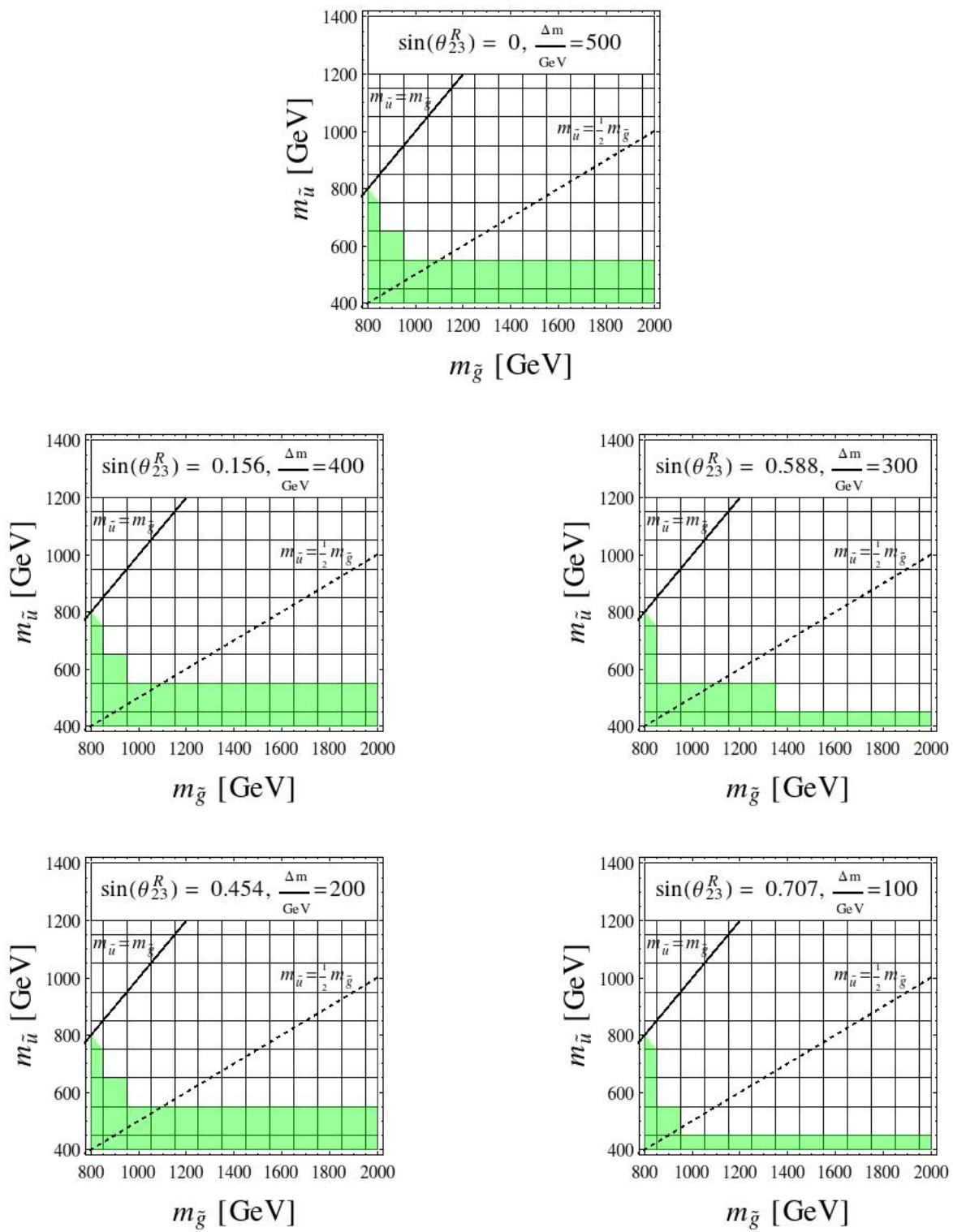

Figure 8. Sensitivity of the ATLAS charm-squark search of ref. [41] for different values of the $\Delta m$ and $\theta_{23}^{R}$ parameters (the exact values being indicated in the top bar of each subfigure). The excluded regions are shown in green in the $\left(m_{\tilde{u}}, m_{\tilde{g}}\right)$ plane, where $m_{\tilde{u}}$ is the mass of the lightest squark (a scharm-like squark here since $\Delta m>0$ ). The upper panel describes the reference scenario in which the lightest squark is a pure scharm state and the stop is almost decoupled.

other, keeping the same color-coding as for the individual results. We present the results in figure 9 and figure 10.

Neglecting the effect of the LSP mass (taken here to be at its preferred value for increasing the missing energy), the main result of the combined reach figures is that the CMS search for three or more jets plus missing energy and zero leptons, CMS-SUS-13012 [40], leads to a robust lower bound on the gluino mass of about $1.2 \mathrm{TeV}$ regardless of the amount of flavor mixing. Where the flavor mixing becomes significant, for $\theta_{23}^{R} \gtrsim \pi / 6$, 

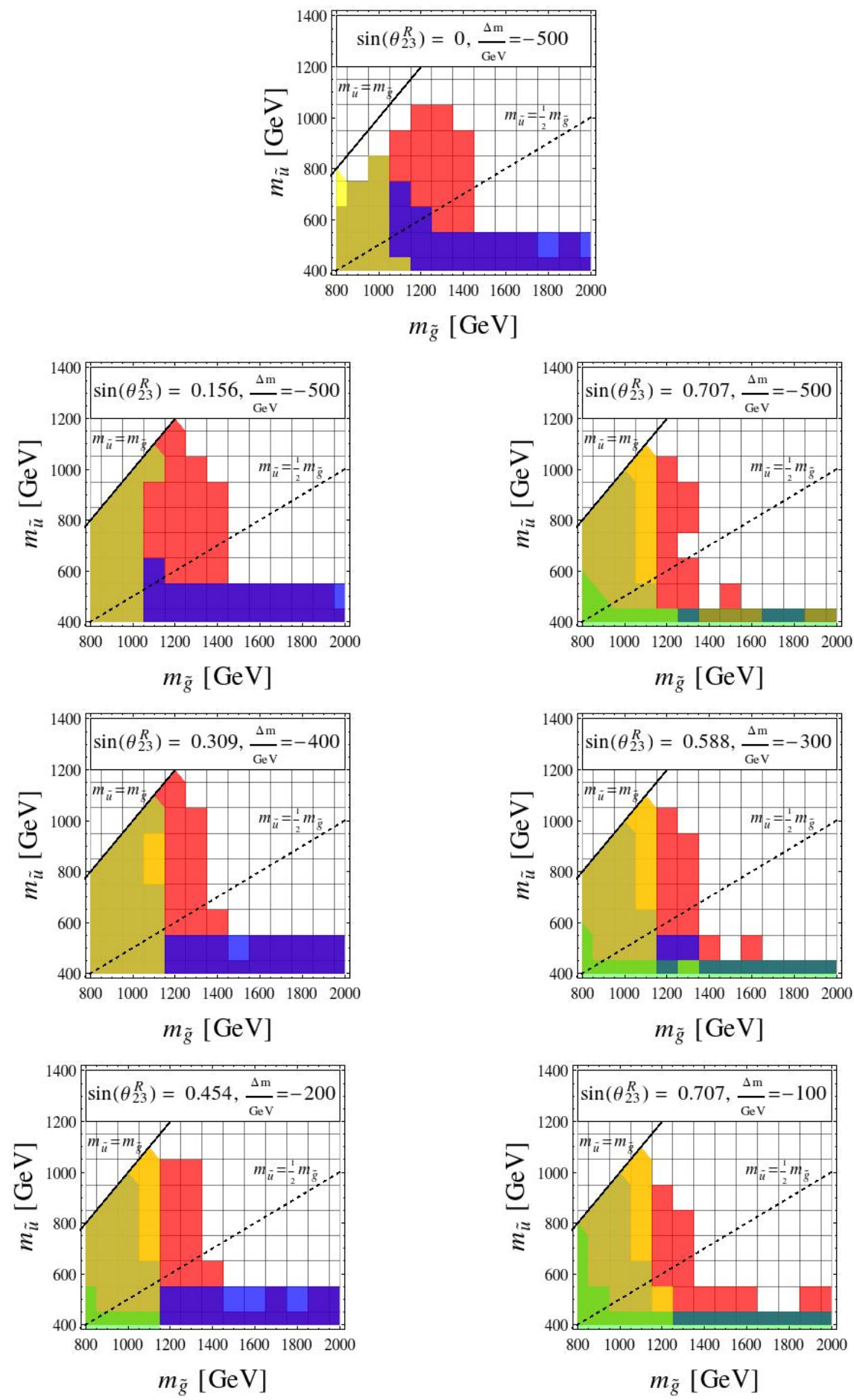

Figure 9. Sensitivity of all four searches pursued in this work for different values of the $\Delta m$ and $\theta_{23}^{R}$ parameters (the exact values being indicated in the top bar of each subfigure). The excluded regions related to the ATLAS-SUSY-2013-04, CMS-SUS-13-011, CMS-SUS-13-012 and ATLASSUSY-2014-03 are respectively shown in red, blue, yellow and green in the $\left(m_{\tilde{u}}, m_{\tilde{g}}\right)$ plane, where $m_{\tilde{u}}$ is the mass of the lightest squark (a stop-like squark here since $\Delta m<0$ ). The upper panel describes the reference scenario in which the lightest squark is a pure stop state and the scharm is almost decoupled. 

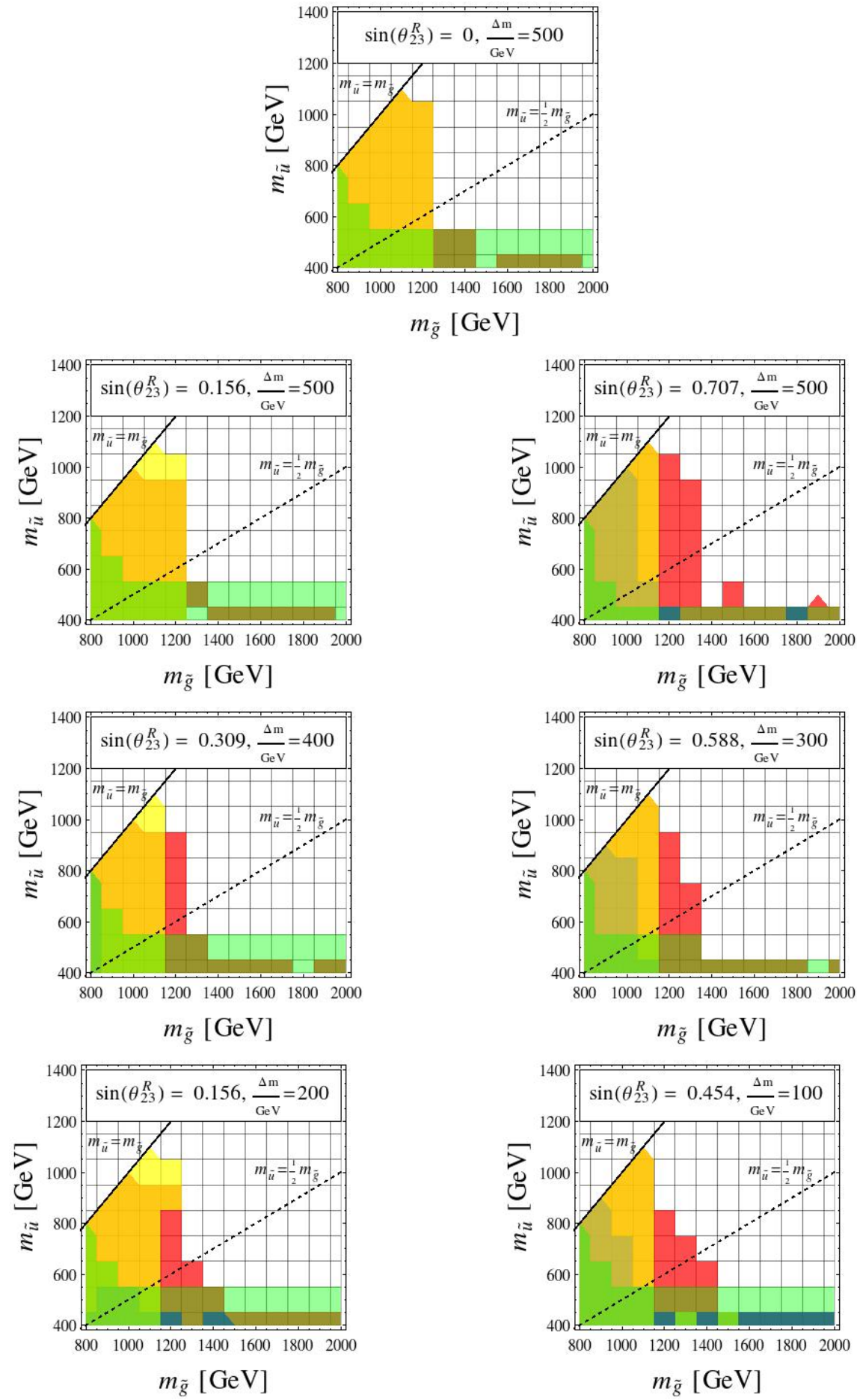

Figure 10. Sensitivity of all four searches pursued in this work for different values of the $\Delta m$ and $\theta_{23}^{R}$ parameters (the exact values being indicated in the top bar of each subfigure). The excluded regions related to the ATLAS-SUSY-2013-04, CMS-SUS-13-011, CMS-SUS-13-012 and ATLASSUSY-2014-03 are respectively shown in red, blue, yellow and green in the $\left(m_{\tilde{u}}, m_{\tilde{g}}\right)$ plane, where $m_{\tilde{u}}$ is the mass of the lightest squark (a scharm-like squark here since $\Delta m>0$ ). The upper panel describes the reference scenario in which the lightest squark is a pure scharm state and the stop is almost decoupled. 
an improvement in the reach is implied by the ATLAS search in the multijet plus missing energy final state, ATLAS-SUSY-2013-04 [39], which leads to even stronger constraints on the squark and gluino masses. Another noteworthy point is that the bound on the lightest squark mass is always greater than about $400 \mathrm{GeV}$, reaching even roughly $600 \mathrm{GeV}$ when this squark is primarily a scharm. Finally, the CMS stop search in the single lepton final state, CMS-SUS-13-011 [38], is only relevant for stop-like lightest squark while for the inverse scenario with a scharm-like lightest squark, the same reach in the parameter space is this time covered roughly by the ATLAS scharm-pair search, ATLAS-SUSY-2014-03 [41].

\section{Conclusions}

In this work we have studied the LHC constraints on the gluino and squark masses in the presence of squark flavor violation. The violation of the flavor symmetry has manifested itself in two interesting and distinct manners. First, we have not assumed squark degeneracy, and in particular, we have considered the case where the squarks of the first and second generations have different masses. Effectively, the first generation squarks, which are subject to the strongest LHC constraints have been taken decoupled, while the would-be (right-handed) scharm eigenstate has been allowed to be significantly lighter. Second, we have allowed the squarks of the second and third generations, in particular the (right-handed) scharm and stop, to mix. Such a scenario has been studied in the past, however, either under the assumption that the gluino is decoupled and/or when the gluino flavor violating couplings are vanishing. In the absence of non-MSSM structures, the former assumption is unnatural, and the latter is inconsistent with the basic flavor structure described above.

We can distinguish between two different sources of tuning. The first type is associated with the contribution of the would-be stop flavor eigenstate to the Higgs-boson mass. In a previous work, this has been studied in models featuring a decoupled gluino [16], with the conclusion that the mixing could slightly improve the level of required tuning. On this front, we have nothing qualitatively new to add beyond the fact that the bounds on the stopand scharm-like states have been slightly improved by the LHC experiments. The second type of tuning is related to the contributions of the gluino to the squark masses, where naturalness requires the gluino mass to be smaller than about twice of that of the squarks. This is particularly relevant to the above study due to the fact that the second generation squark masses are less constrained by the LHC searches. Thus, the main purpose of our study has been to examine what are the bounds on the gluino-squark system within the above framework.

As the combined reach of the four LHC analyses that we have considered has shown, the fine-tuning requirement still allows for sizable stop-scharm mixing. Equivalently, we have found that for various values of the mixing, there is a wide range of unexcluded and relatively light squark and gluino masses which satisfy the gluino-mass naturalness criterion. In fact, for some mixings and mass splitting cases, the 'natural' region in the squark-gluino mass plane could even become larger. 
In the foreseen future, the experiments are expected to publish new results with an improved reach. An improvement in the sensitivity to the above framework is obviously expected due to the increase in the center of mass energy. Furthermore, as the ATLAS collaboration has now effectively installed a new inner layer of pixel detector (IBL), its charm-tagging capabilities are expected to be upgraded, resulting in more efficient ways to look for charm squarks. Moreover, we note that during the finalizing of this work, CMS has released several analyses which target stop pair production and which are likely sensitive to gluinos as well. The search with highest reach is presented in ref. [66], with stop masses excluded up to roughly $750 \mathrm{GeV}$ (for an LSP defined as in our model). Such an improved reach would cover additional parameter-space in our gluino-squark mass plane, however, it is expected to exhibit similar characteristics once the mixing and mass-splittings are turned on. As a result, the new CMS results stress the importance of flavor even beyond the reach shown in this work.

Finally, we point out that we have not discussed here the impact of flavor violation on low energy observables. We have also ignored the bounds coming from the Higgsino sector even though naturalness suggests that those should be at the bottom of the supersymmetric spectrum modulo, possibly, the dark matter candidate itself. These are beyond the scope of our study.

\section{Acknowledgments}

The authors are extremely grateful to David Cohen, the administrator of the ATLASTechnion grid project for his extensive support. His personal support, and assignment of resources have greatly facilitated in the progress of this work. We also thank Monica d'Onofrio and Tomasso Lari for their help with the reimplementation of the ATLASSUSY-2013-04 search. BF is supported by the Theory-LHC-France initiative of the CNRS (INP/IN2P3). IG is supported by NSF grant PHY-1316792. The work of GP is supported by BSF, ERC and ISF grants.

\section{A Implementation and validation of ATLAS-SUSY-2013-04 in MAD- ANALYSIS 5}

The recast of the ATLAS-SUSY-2013-04 search makes use of the MADANALYSIS 5 framework together with the MA5TUNE version of DeLPHES described in ref. [42]. Our implementation therefore relies on the interfacing of DELPHES as implemented in the version 1.1.12 of MadAnalysis 5. The necessary configuration file of Delphes can be found on the public analysis database webpage,

http://madanalysis.irmp.ucl.ac.be/wiki/PublicAnalysisDatabase .

The validation of the analysis implementation has been achieved by relying on a set of benchmark scenarios belonging to the gluino-stop (off-shell) model introduced in the ATLAS publication [39]. In this case, the SM is supplemented by a gluino and a neutralino, and the gluino is enforced to decay into a top-antitop final state via an off-shell top squark. 


\begin{tabular}{|l|c|c|c|c|}
\hline \multirow{2}{*}{ Selection step } & \multicolumn{2}{|c|}{ Events counts } & \multicolumn{2}{c|}{ Relative change } \\
& MADANALYSIS 5 & ATLAS & MADANALYSI 5 & ATLAS \\
\hline Initial number of events & 206.3 & 206.3 & & \\
6 jets with $E_{T}>45 \mathrm{GeV}$ & 157.0 & 168 & $-23.9 \%$ & $-18.6 \%$ \\
lepton veto & 88.1 & 78 & $-43.9 \%$ & $-53.6 \%$ \\
\hline 8 jets $\left(p_{T}>50 \mathrm{GeV}\right)$ & 18.3 & 16.3 & $-79.2 \%$ & $-79.1 \%$ \\
$\mathbb{E}_{T} / \sqrt{H_{T}}>4 \mathrm{GeV}^{1 / 2}$ & 15.4 & 14.1 & $-15.8 \%$ & $-13.5 \%$ \\
$\rightarrow$ without $b$-tags & 0.97 & 0.85 & $-93.7 \%$ & $-94.0 \%$ \\
$\rightarrow$ with $1 b$-tag & 3.80 & 3 & $-75.3 \%$ & $-78.7 \%$ \\
$\rightarrow$ with $2 b$-tags & 10.7 & 11 & $-30.5 \%$ & $-22.0 \%$ \\
\hline 9 jets $\left(p_{T}>50 \mathrm{GeV}\right)$ & 11.6 & 9.6 & $-86.8 \%$ & $-87.7 \%$ \\
$\mathbb{E}_{T} / \sqrt{H_{T}}>4 \mathrm{GeV} / 2$ & 9.32 & 8.0 & $-19.7 \%$ & $-16.7 \%$ \\
$\rightarrow$ without $b$-tags & 0.70 & 0.33 & $-92.5 \%$ & $-95.9 \%$ \\
$\rightarrow$ with $1 b$-tag & 1.93 & 1.7 & $-79.3 \%$ & $-78.8 \%$ \\
$\rightarrow$ with $2 b$-tags & 6.68 & 6.5 & $-28.3 \%$ & $-18.8 \%$ \\
\hline$\geq 10$ jets $\left(p_{T}>50 \mathrm{GeV}\right)$ & 6.99 & 5.7 & $-92.1 \%$ & $-92.7 \%$ \\
$\mathbb{E}_{T} / \sqrt{H_{T}}>4 \mathrm{GeV}{ }^{1 / 2}$ & 5.34 & 4.7 & $-23.6 \%$ & $-17.5 \%$ \\
\hline 7 jets $\left(p_{T}>80 \mathrm{GeV}\right)$ & 9.72 & 7.53 & $-88.1 \%$ & $-87.7 \%$ \\
$\mathbb{E}_{T} / \sqrt{H_{T}}>4 \mathrm{GeV}^{1 / 2}$ & 8.07 & 6.25 & $-17.0 \%$ & $-17.0 \%$ \\
$\rightarrow$ without $b$-tags & 0.47 & 0.31 & $-94.2 \%$ & $-95.0 \%$ \\
$\rightarrow$ with $1 b$-tag & 1.73 & 1.3 & $-78.6 \%$ & $-79.2 \%$ \\
$\rightarrow$ with $2 b$-tags & 5.86 & 5.1 & $-27.4 \%$ & $-18.4 \%$ \\
\hline$\geq 8$ jets $\left(p_{T}>80 \mathrm{GeV}\right)$ & 4.72 & 3.2 & $-94.6 \%$ & $-95.9 \%$ \\
$\mathbb{E}_{T} / \sqrt{H_{T}}>4 \mathrm{GeV}{ }^{1 / 2}$ & 3.65 & 2.6 & $-22.7 \%$ & $-18.8 \%$ \\
$\rightarrow$ without $b$-tags & 0.06 & 0.13 & $-98.4 \%$ & $-95.0 \%$ \\
$\rightarrow$ with $1 b$-tag & 0.72 & 0.55 & $-80.3 \%$ & $-78.8 \%$ \\
$\rightarrow$ with $2 b$-tags & 2.87 & 2.1 & $-21.4 \%$ & $-19.2 \%$ \\
\hline
\end{tabular}

Table 2. Summary of yields for a gluino-stop off-shell scenario in which the gluino and neutralino masses have been fixed to 1100 and $400 \mathrm{GeV}$, respectively. The results obtained with MADANALYSIS 5 are compared to the official ATLAS results, both in terms of event counts and efficiencies computed from the number of events before and after each of the selection steps.

For validation purposes, we generate events following the procedure of the ATLAS collaboration, using the HeRwIG++ program [67] for the simulation of the hard process, the parton showering and the hadronization. The supersymmetric spectrum file has been provided by the ATLAS collaboration via HEPDATA and the HERWIG++ configuration that we have used can be obtained from the MADANALYsis 5 webpage.

In table 2, we compare the ATLAS results for the cut-flow counts to those obtained with our reimplementation of the ATLAS-SUSY-2013-04 analysis in MADANALYSIS 5. We present the surviving number of events after each step of the selection strategy for the 


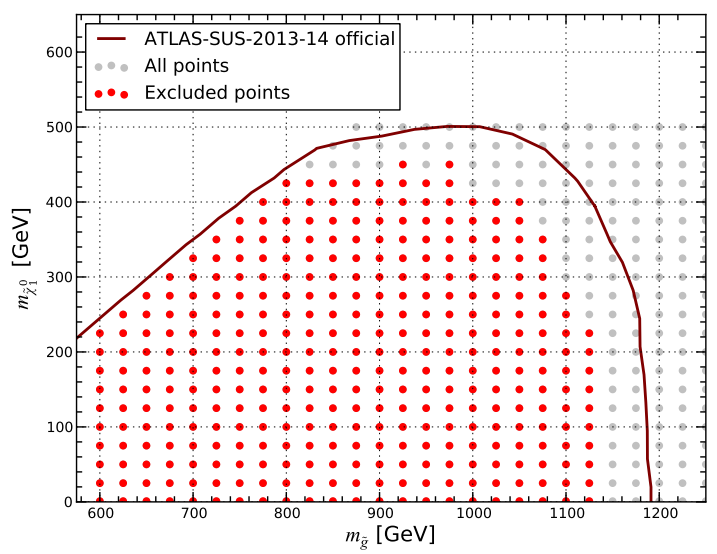

Figure 11. 95\% CL exclusion limit for gluino-stop off-shell scenarios presented as contours in the gluino-neutralino mass plane. We compare results obtained with MADANALYsis 5 (dots) to the official ATLAS results (solid line).

13 signal regions under consideration and for a scenario in which the gluino mass is set to $1100 \mathrm{GeV}$ and the neutralino mass to $400 \mathrm{GeV}$. We have found that all selection steps are properly described by the MADANALYSIS 5 implementation, the agreement reaching the level of about $10 \%$. In figure 11, we move away from the chosen benchmark scenario and vary the gluino and neutralino masses freely, enforcing however that the gluino decay channel into a top-antitop pair and a neutralino stays open. We observe that our machinery allows us to reproduce the ATLAS official bounds (obtained from HEPDATA) at the $50 \mathrm{GeV}$ level, which is acceptable on the basis of the limitations of our procedure mentioned in section 3.1. The MADANALYSIS 5 implementation of the analysis can be obtained from INSPIRE [68].

Open Access. This article is distributed under the terms of the Creative Commons Attribution License (CC-BY 4.0), which permits any use, distribution and reproduction in any medium, provided the original author(s) and source are credited.

\section{References}

[1] ATLAS collaboration, Observation of a new particle in the search for the standard model Higgs boson with the ATLAS detector at the LHC, Phys. Lett. B 716 (2012) 1 [arXiv: 1207.7214] [INSPIRE].

[2] CMS collaboration, Observation of a new boson at a mass of $125 \mathrm{GeV}$ with the CMS experiment at the LHC, Phys. Lett. B 716 (2012) 30 [arXiv:1207.7235] [INSPIRE].

[3] ATLAS and CMS Collaborations, Measurements of the Higgs boson production and decay rates and constraints on its couplings from a combined ATLAS and CMS analysis of the LHC pp collision data at $\sqrt{s}=7$ and 8 TeV, ATLAS-CONF-2015-044 (2015).

[4] LHCb collaboration, Observation of $J / \psi p$ resonances consistent with pentaquark states in $\lambda_{b}^{0} \rightarrow J / \psi K^{-} p$ decays, Phys. Rev. Lett. 115 (2015) 072001 [arXiv:1507.03414] [InSPIRE].

[5] LHCb collaboration, Differential branching fractions and isospin asymmetries of $B \rightarrow K^{(*)} \mu^{+} \mu^{-}$decays, JHEP 06 (2014) 133 [arXiv: 1403. 8044] [INSPIRE]. 
[6] LHCb collaboration, Test of lepton universality using $B^{+} \rightarrow K^{+} \ell^{+} \ell^{-}$decays, Phys. Rev. Lett. 113 (2014) 151601 [arXiv: 1406.6482] [INSPIRE].

[7] LHCb collaboration, Measurement of the ratio of branching fractions $\mathcal{B}\left(\bar{B}^{0} \rightarrow D^{*+} \tau^{-} \bar{\nu}_{\tau}\right) / \mathcal{B}\left(\bar{B}^{0} \rightarrow D^{*+} \mu^{-} \bar{\nu}_{\mu}\right)$, Phys. Rev. Lett. 115 (2015) 111803 [arXiv: 1506.08614] [INSPIRE].

[8] G.F. Giudice, Naturalness after LHC8, PoS (EPS-HEP 2013) 163 [arXiv:1307.7879] [INSPIRE].

[9] J.L. Feng, Naturalness and the status of supersymmetry, Ann. Rev. Nucl. Part. Sci. 63 (2013) 351 [arXiv:1302.6587] [INSPIRE].

[10] O. Gedalia, J.F. Kamenik, Z. Ligeti and G. Perez, On the universality of CP-violation in $\Delta F=1$ processes, Phys. Lett. B 714 (2012) 55 [arXiv:1202.5038] [INSPIRE].

[11] Y. Nir and N. Seiberg, Should squarks be degenerate?, Phys. Lett. B 309 (1993) 337 [hep-ph/9304307] [INSPIRE].

[12] I. Galon, G. Perez and Y. Shadmi, Non-degenerate squarks from flavored gauge mediation, JHEP 09 (2013) 117 [arXiv: 1306.6631] [INSPIRE].

[13] C. Delaunay, O. Gedalia, S.J. Lee, G. Perez and E. Ponton, Ultra visible warped model from flavor triviality and improved naturalness, Phys. Rev. D 83 (2011) 115003 [arXiv: 1007.0243] [INSPIRE].

[14] A. Azatov, G. Panico, G. Perez and Y. Soreq, On the flavor structure of natural composite higgs models \& top flavor violation, JHEP 12 (2014) 082 [arXiv: 1408.4525] [INSPIRE].

[15] R. Mahbubani, M. Papucci, G. Perez, J.T. Ruderman and A. Weiler, Light nondegenerate squarks at the LHC, Phys. Rev. Lett. 110 (2013) 151804 [arXiv:1212.3328] [INSPIRE].

[16] M. Blanke, G.F. Giudice, P. Paradisi, G. Perez and J. Zupan, Flavoured naturalness, JHEP 06 (2013) 022 [arXiv: 1302.7232] [INSPIRE].

[17] S. Heinemeyer, W. Hollik, F. Merz and S. Penaranda, Electroweak precision observables in the MSSM with nonminimal flavor violation, Eur. Phys. J. C 37 (2004) 481 [hep-ph/0403228] [INSPIRE].

[18] J. Cao, G. Eilam, K.-i. Hikasa and J.M. Yang, Experimental constraints on stop-scharm flavor mixing and implications in top-quark FCNC processes, Phys. Rev. D 74 (2006) 031701 [hep-ph/0604163] [INSPIRE].

[19] G. Bozzi, B. Fuks, B. Herrmann and M. Klasen, Squark and gaugino hadroproduction and decays in non-minimal flavour violating supersymmetry, Nucl. Phys. B 787 (2007) 1 [arXiv: 0704.1826] [INSPIRE].

[20] M. Artuso et al., B, D and K decays, Eur. Phys. J. C 57 (2008) 309 [arXiv:0801.1833] [INSPIRE].

[21] B. Fuks, B. Herrmann and M. Klasen, Flavour violation in gauge-mediated supersymmetry breaking models: experimental constraints and phenomenology at the LHC, Nucl. Phys. B 810 (2009) 266 [arXiv:0808.1104] [INSPIRE].

[22] T. Hurth and W. Porod, Flavour violating squark and gluino decays, JHEP 08 (2009) 087 [arXiv: 0904.4574] [INSPIRE].

[23] B. Fuks, B. Herrmann and M. Klasen, Phenomenology of anomaly-mediated supersymmetry 
breaking scenarios with non-minimal flavour violation, Phys. Rev. D 86 (2012) 015002

[arXiv: 1112.4838] [INSPIRE].

[24] P. Agrawal and C. Frugiuele, Mixing stops at the LHC, JHEP 01 (2014) 115 [arXiv: 1304.3068] [INSPIRE].

[25] M. Arana-Catania, S. Heinemeyer and M.J. Herrero, Updated constraints on general squark flavor mixing, Phys. Rev. D 90 (2014) 075003 [arXiv:1405.6960] [inSPIRE].

[26] K. Kowalska, Phenomenology of SUSY with general flavour violation, JHEP 09 (2014) 139 [arXiv:1406.0710] [INSPIRE].

[27] M. Backović, A. Mariotti and M. Spannowsky, Signs of tops from highly mixed stops, JHEP 06 (2015) 122 [arXiv:1504.00927] [INSPIRE].

[28] K. De Causmaecker et al., General squark flavour mixing: constraints, phenomenology and benchmarks, JHEP 11 (2015) 125 [arXiv:1509.05414] [INSPIRE].

[29] C. Brust, A. Katz, S. Lawrence and R. Sundrum, SUSY, the third generation and the LHC, JHEP 03 (2012) 103 [arXiv: 1110.6670] [INSPIRE].

[30] T. Han, K.-i. Hikasa, J.M. Yang and X.-m. Zhang, The FCNC top squark decay as a probe of squark mixing, Phys. Rev. D 70 (2004) 055001 [hep-ph/0312129] [INSPIRE].

[31] G. Hiller and Y. Nir, Measuring flavor mixing with minimal flavor violation at the LHC, JHEP 03 (2008) 046 [arXiv: 0802.0916] [INSPIRE].

[32] G.D. Kribs, A. Martin and T.S. Roy, Squark flavor violation at the LHC, JHEP 06 (2009) 042 [arXiv:0901.4105] [INSPIRE].

[33] A. Bartl et al., Impact of squark generation mixing on the search for gluinos at LHC, Phys. Lett. B 679 (2009) 260 [arXiv:0905.0132] [INSPIRE].

[34] A. Bartl, H. Eberl, B. Herrmann, K. Hidaka, W. Majerotto and W. Porod, Impact of squark generation mixing on the search for squarks decaying into fermions at LHC, Phys. Lett. B 698 (2011) 380 [Erratum ibid. B 700 (2011) 390] [arXiv: 1007.5483] [INSPIRE].

[35] M. Bruhnke, B. Herrmann and W. Porod, Signatures of bosonic squark decays in non-minimally flavour-violating supersymmetry, JHEP 09 (2010) 006 [arXiv:1007.2100] [INSPIRE].

[36] A. Bartl et al., Flavour violating gluino three-body decays at LHC, Phys. Rev. D 84 (2011) 115026 [arXiv: 1107.2775] [INSPIRE].

[37] A. Bartl et al., Flavor violating bosonic squark decays at LHC, Int. J. Mod. Phys. A 29 (2014) 1450035 [arXiv: 1212.4688] [INSPIRE].

[38] CMS collaboration, Search for top-squark pair production in the single-lepton final state in pp collisions at $\sqrt{s}=8 \mathrm{TeV}$, Eur. Phys. J. C 73 (2013) 2677 [arXiv:1308.1586] [InSPIRE].

[39] ATLAS collaboration, Search for new phenomena in final states with large jet multiplicities and missing transverse momentum at $\sqrt{s}=8$ TeV proton-proton collisions using the ATLAS experiment, JHEP 10 (2013) 130 [Erratum ibid. 01 (2014) 109] [arXiv:1308.1841] [INSPIRE].

[40] CMS collaboration, Search for new physics in the multijet and missing transverse momentum final state in proton-proton collisions at $\sqrt{s}=8 \mathrm{TeV}$, JHEP 06 (2014) 055 [arXiv:1402.4770] [INSPIRE]. 
[41] ATLAS collaboration, Search for scalar charm quark pair production in pp collisions at $\sqrt{s}=8 \mathrm{TeV}$ with the ATLAS detector, Phys. Rev. Lett. 114 (2015) 161801 [arXiv: 1501.01325] [INSPIRE].

[42] B. Dumont et al., Toward a public analysis database for LHC new physics searches using MADANALYSIS 5, Eur. Phys. J. C 75 (2015) 56 [arXiv:1407.3278] [inSPIRE].

[43] J. Alwall, P. Schuster and N. Toro, Simplified models for a first characterization of new physics at the LHC, Phys. Rev. D 79 (2009) 075020 [arXiv:0810.3921] [INSPIRE].

[44] LHC New Physics Working Group collaboration, D. Alves, Simplified models for LHC new physics searches, J. Phys. G 39 (2012) 105005 [arXiv:1105.2838] [InSPIRE].

[45] G. Raz, The Mass insertion approximation without squark degeneracy, Phys. Rev. D 66 (2002) 037701 [hep-ph/0205310] [INSPIRE].

[46] Y. Nir and G. Raz, Quark squark alignment revisited, Phys. Rev. D 66 (2002) 035007 [hep-ph/0206064] [INSPIRE].

[47] M. Ciuchini, A. Masiero, P. Paradisi, L. Silvestrini, S.K. Vempati and O. Vives, Soft SUSY breaking grand unification: leptons versus quarks on the flavor playground, Nucl. Phys. B 783 (2007) 112 [hep-ph/0702144] [INSPIRE].

[48] G. Isidori, Y. Nir and G. Perez, Flavor physics constraints for physics beyond the standard model, Ann. Rev. Nucl. Part. Sci. 60 (2010) 355 [arXiv: 1002.0900].

[49] L. Calibbi, I. Galon, A. Masiero, P. Paradisi and Y. Shadmi, Charged slepton flavor post the $8 \mathrm{TeV}$ LHC: a simplified model analysis of low-energy constraints and LHC SUSY searches, JHEP 10 (2015) 043 [arXiv:1502.07753] [INSPIRE].

[50] A. Alloul, N.D. Christensen, C. Degrande, C. Duhr and B. Fuks, FeynRules 2.0 - A complete toolbox for tree-level phenomenology, Comput. Phys. Commun. 185 (2014) 2250 [arXiv: 1310.1921] [INSPIRE].

[51] C. Degrande, C. Duhr, B. Fuks, D. Grellscheid, O. Mattelaer and T. Reiter, UFO - The Universal FeynRules Output, Comput. Phys. Commun. 183 (2012) 1201 [arXiv:1108.2040] [INSPIRE].

[52] J. Alwall et al., The automated computation of tree-level and next-to-leading order differential cross sections and their matching to parton shower simulations, JHEP 07 (2014) 079 [arXiv: 1405.0301] [INSPIRE].

[53] T. Sjöstrand, S. Mrenna and P.Z. Skands, PYTHIA 6.4 physics and manual, JHEP 05 (2006) 026 [hep-ph/0603175] [INSPIRE].

[54] DELPHES 3 collaboration, J. de Favereau et al., DELPHES 3, a modular framework for fast simulation of a generic collider experiment, JHEP 02 (2014) 057 [arXiv:1307.6346] [INSPIRE].

[55] M. Cacciari, G.P. Salam and G. Soyez, The anti-k $k_{t}$ jet clustering algorithm, JHEP 04 (2008) 063 [arXiv: 0802.1189] [INSPIRE].

[56] M. Cacciari, G.P. Salam and G. Soyez, FastJet user manual, Eur. Phys. J. C 72 (2012) 1896 [arXiv:1111.6097] [INSPIRE].

[57] E. Conte, B. Fuks and G. Serret, MadAnalysis 5, a user-friendly framework for collider phenomenology, Comput. Phys. Commun. 184 (2013) 222 [arXiv:1206.1599] [INSPIRE]. 
[58] E. Conte, B. Dumont, B. Fuks and C. Wymant, Designing and recasting LHC analyses with MadAnalysis 5, Eur. Phys. J. C 74 (2014) 3103 [arXiv:1405.3982] [INSPIRE].

[59] A.L. Read, Presentation of search results: the CL(s) technique, J. Phys. G 28 (2002) 2693 [INSPIRE].

[60] M.L. Mangano, M. Moretti, F. Piccinini and M. Treccani, Matching matrix elements and shower evolution for top-quark production in hadronic collisions, JHEP 01 (2007) 013 [hep-ph/0611129] [INSPIRE].

[61] J. Alwall, S. de Visscher and F. Maltoni, QCD radiation in the production of heavy colored particles at the LHC, JHEP 02 (2009) 017 [arXiv:0810.5350] [INSPIRE].

[62] Y. Bai, H.-C. Cheng, J. Gallicchio and J. Gu, Stop the top background of the stop search, JHEP 07 (2012) 110 [arXiv:1203.4813] [INSPIRE].

[63] B. Dumont, B. Fuks and C. Wymant, Search for top-squark pair production in the single-lepton final state in pp collisions at $\sqrt{s}=8$ TeV, Eur. Phys. J. C 73 (2013) 2677 [arXiv: 1308.1586] [CMS-SUS-13-011] [INSPIRE].

[64] S. Bein and D. Sengupta, Search for new physics in the multijet and missing transverse momentum final state in proton-proton collisions at $\sqrt{s}=8 \mathrm{TeV}$, JHEP 06 (2014) 055 [arXiv: 1402.4770] [CMS-SUS-13-012] [INSPIRE].

[65] D.R. Tovey, On measuring the masses of pair-produced semi-invisibly decaying particles at hadron colliders, JHEP 04 (2008) 034 [arXiv: 0802 . 2879] [INSPIRE].

[66] CMS collaboration, A search for scalar top quark production and decay to all hadronic final states in pp collisions at $\sqrt{s}=8 \mathrm{TeV}$, CMS-PAS-SUS-13-023 [INSPIRE].

[67] M. Bahr et al., HERWIG++ physics and manual, Eur. Phys. J. C 58 (2008) 639 [arXiv:0803.0883] [INSPIRE].

[68] B. Fuks, M. Blanke and I. Galon, MadAnalysis 5 implementation of ATLAS-SUSY-2013-04 [INSPIRE]. 